\title{
Maximality Principle and General Results of Ekeland and Caristi Types without Lower Semicontinuity Assumptions in Cone Uniform Spaces with Generalized Pseudodistances
}

\author{
Kazimierz Włodarczyk and Robert Plebaniak \\ Department of Nonlinear Analysis, Faculty of Mathematics and Computer Science, University of Łódz, \\ Banacha 22, 90-238 Łódź, Poland \\ Correspondence should be addressed to Kazimierz Włodarczyk, wlkzxa@math.uni.lodz.pl \\ Received 31 December 2009; Accepted 8 March 2010 \\ Academic Editor: Tomonari Suzuki
}

Copyright (C 2010 K. Włodarczyk and R. Plebaniak. This is an open access article distributed under the Creative Commons Attribution License, which permits unrestricted use, distribution, and reproduction in any medium, provided the original work is properly cited.

\begin{abstract}
Our aim is twofold: first, we want to introduce a partial quasiordering in cone uniform spaces with generalized pseudodistances for giving the general maximality principle in these spaces. Second, we want to show how this maximality principle can be used to obtain new and general results of Ekeland and Caristi types without lower semicontinuity assumptions, which was not done in the previous publications on this subject.
\end{abstract}

\section{Introduction}

The famous Banach contraction principle [1], fundamental in fixed point theory, has been extended in many different directions. Among these extensions, Caristi's fixed point theorem [2] concerning dissipative maps with lower semicontinuous entropies, equivalent to celebrated Ekeland's variational principle [3] providing approximate solutions of nonconvex minimization problems concerning lower semicontinuous maps, may be the most valuable one.

These results are very useful, simple, and important tools for investigating various problems in nonlinear analysis, mathematical programming, control theory, abstract economy, global analysis, and others. They have many generalizations and extensive applications in many fields of mathematics and applied mathematics.

In the literature, the several generalizations of the variational principle of Ekeland type, for lower semicontinuous maps and fixed point and endpoint theorem of Caristi type for dissipative single-valued and set-valued dynamic systems with lower semicontinuous entropies in metric and uniform spaces are given, and various techniques and methods of 
investigations (notably based on maximality principle) are presented. However, in all these papers the restrictive assumptions about lower semicontinuity are essential. For details see [4-29] and references therein. It is not our purpose to give a complete list of related papers here.

A long time ago, we did not know how to define the distances in metric, uniform, or cone uniform spaces, which generalize metrics, pseudometrics, or cone pseudometrics, which are connected with metrics, pseudometrics, or cone pseudometrics, respectively, and which have applications to obtaining the solutions of several new important problems in nonlinear analysis. The pioneering effort in this direction is papers of Tataru [30] in Banach spaces,Kada et al. [31], Suzuki [32], and Lin and Du [33] in metric spaces, and Vályi [34] in uniform spaces. In these papers, among other things, various distances are introduced, and relations between Tataru [30], and Kada et al. [31] distances and distances of Suzuki [32] and Lin and Du [33] are established. For many applications of these distances, see the papers [30-48] where, among other things, in metric and uniform spaces with generalized distances [30-34], the new fixed point theorems of Caristi' type for dissipative maps with lower semicontinuous entropies and variational principles of Ekeland type for lower semicontinuous maps are given.

In this paper, in cone uniform spaces $[49,50]$, the families of generalized pseudodistances are introduced (see Section 2), a partial quasiordering is defined and the general maximality principle is formulated and proved (see Section 3). As applications, in cone uniform spaces with the families of generalized pseudodistances, the general variational principle of Ekeland type for not necessarily lower semicontinuous maps and a fixed point and endpoint theorem of Caristi type for dissipative set-valued dynamic systems with not necessarily lower semicontinuous entropies are established (see Section 4). Special cases are discussed and examples and comparisons show a fundamental difference between our results and the well-known ones in the literature where the standard lower semicontinuity assumptions are essential (see Section 5). Relations between our generalized pseudodistances and generalized distances are described (see Section 6; the aim of this section is to prove that each generalized distance [30-34] is a generalized pseudodistance and we construct the examples which show that the converse is not true). The definitions, the results, the ideas and the methods presented here are new for set-valued and single-valued dynamic systems in cone uniform, cone locally convex and cone metric spaces and even in uniform, locally convex, and metric spaces.

\section{Generalized Pseudodistances in Cone Uniform Spaces}

We define a real normed space to be a pair $(L,\|\cdot\|)$, with the understanding that a vector space $L$ over $\mathbb{R}$ carries the topology generated by the metric $(a, b) \rightarrow\|a-b\|, a, b \in L$.

Let $L$ be a real normed space. A nonempty closed convex set $H \subset L$ is called a cone in $L$ if it satisfies (H1) $\forall_{s \in(0, \infty)}\{s H \subset H\},(\mathbf{H} 2) H \cap(-H)=\{0\}$, and (H3) $H \neq\{0\}$.

It is clear that each cone $H \subset L$ defines, by virtue of " $a \preceq_{H} b$ if and only if $b-a \in H$ ", an order of $L$ under which $L$ is an ordered normed space with cone $H$. We will write $a \prec_{H} b$ to indicate that $a \leq_{H} b$ but $a \neq b$.

A cone $H$ is said to be solid if $\operatorname{int}(H) \neq \emptyset$; $\operatorname{int}(H)$ denotes the interior of $H$. We will write $a \ll b$ to indicate that $b-a \in \operatorname{int}(H)$.

The cone $H$ is normal if a real number $M>0$ exists such that for each $a, b \in H$, $0 \preceq_{H} a \preceq_{H} b$ implies that $\|a\| \leqslant M\|b\|$. The number $M$ satisfying the above is called the normal constant of $H$. 
The following terminologies will be much used.

Definition 2.1 (see $[49,50]$ ). Let $X$ be a nonempty set and let $L$ be an ordered normed space with cone $H$.

(i) The family $D=\left\{p_{\alpha}: X \times X \rightarrow L, \alpha \in \mathcal{A}\right\}$, A-index set, is said to be a $P$-family of cone pseudometrics on $X(D$-family, for short $)$ if the following three conditions hold:

(D1) $\forall_{\alpha \in \mathscr{A}} \forall_{x, y \in X}\left\{0 \leq_{H} p_{\alpha}(x, y) \wedge x=y \Rightarrow p_{\alpha}(x, y)=0\right\}$;

(D2) $\forall_{\alpha \in \mathscr{A}} \forall_{x, y \in X}\left\{p_{\alpha}(x, y)=p_{\alpha}(y, x)\right\}$

(D3) $\forall_{\alpha \in \mathcal{A}} \forall_{x, y, z \in X}\left\{p_{\alpha}(x, z) \leq_{H} p_{\alpha}(x, y)+p_{\alpha}(y, z)\right\}$.

(ii) If $P$ is a $D$-family, then the pair $(X, D)$ is called a cone uniform space.

(iii) A $p$-family $D$ is said to be separating if

$$
\text { (D4) } \forall_{x, y \in X}\left\{x \neq y \Rightarrow \exists_{\alpha \in \mathcal{A}}\left\{0 \prec_{H} p_{\alpha}(x, y)\right\}\right\} \text {. }
$$

(iv) If a $D$-family $D$ is separating, then the pair $(X, D)$ is called a Hausdorff cone uniform space.

Definition 2.2 (see [49, Definition 2.3]). Let $L$ be an ordered normed space with solid cone $H$ and let $(X, P)$ be a cone uniform space with cone $H$.

(i) We say that a sequence $\left(w_{m}: m \in \mathbb{N}\right)$ in $X$ is a $p$-convergent in $X$, if there exists $w \in X$ such that

$$
\forall_{\alpha \in \mathcal{A}} \forall_{c_{\alpha} \in L, 0 \ll c_{\alpha}} \exists_{n_{0}=n_{0}\left(\alpha, c_{\alpha}\right) \in \mathbb{N}} \forall_{m \in \mathbb{N} ; n_{0} \leqslant m}\left\{p_{\alpha}\left(w_{m}, w\right) \ll c_{\alpha}\right\} .
$$

(ii) We say that a sequence $\left(w_{m}: m \in \mathbb{N}\right)$ in $X$ is a $p$-Cauchy sequence in $X$, if

$$
\forall_{\alpha \in \mathcal{A}} \forall_{\mathcal{C}_{\alpha} \in L, 0 \ll c_{\alpha}} \exists_{n_{0}=n_{0}\left(\alpha, c_{\alpha}\right) \in \mathbb{N}} \forall_{m, n \in \mathbb{N} ; n_{0} \leqslant m<n}\left\{p_{\alpha}\left(w_{m}, w_{n}\right) \ll c_{\alpha}\right\} .
$$

(iii) If every $p$-Cauchy sequence in $X$ is $p$-convergent in $X$, then $(X, p)$ is called a $p$ sequentially complete cone uniform space.

The following holds.

Theorem 2.3 (see [49, Theorem 2.1]). Let $L$ be an ordered normed space with normal solid cone $H$ and let $(X, D)$ be a Hausdorff cone uniform space with cone $H$.

(a) Let $\left(w_{m}: m \in \mathbb{N}\right)$ be a sequence in $X$ and let $w \in X$. The sequence $\left(w_{m}: m \in \mathbb{N}\right)$ is p-convergent to $w$ if and only if

$$
\forall_{\alpha \in \mathscr{A}} \forall_{\varepsilon_{\alpha}>0} \exists_{n_{0}=n_{0}\left(\alpha, \varepsilon_{\alpha}\right) \in \mathbb{N}} \forall_{m \in \mathbb{N} ; n_{0} \leqslant m}\left\{\left\|p_{\alpha}\left(w_{m}, w\right)\right\|<\varepsilon_{\alpha}\right\} .
$$

(b) Let $\left(w_{m}: m \in \mathbb{N}\right)$ be a sequence in $X$. The sequence $\left(w_{m}: m \in \mathbb{N}\right)$ is a $D$-Cauchy sequence if and only if

$$
\forall_{\alpha \in \mathcal{A}} \forall_{\varepsilon_{\alpha}>0} \exists_{n_{0}=n_{0}\left(\alpha, \varepsilon_{\alpha}\right) \in \mathbb{N}} \forall_{m, n \in \mathbb{N} ; n_{0} \leqslant m<n}\left\{\left\|p_{\alpha}\left(w_{m}, w_{n}\right)\right\|<\varepsilon_{\alpha}\right\} .
$$

(c) Each $D$-convergent sequence is a $D$-Cauchy sequence. 
Fixed Point Theory and Applications

Definition 2.4. Let $L$ be an ordered normed space with solid cone $H$. The cone $H$ is called regular if for every increasing (decreasing) sequence which is bounded from above (below), that is, if for each sequence $\left(c_{m}: m \in \mathbb{N}\right)$ in $L$ such that $c_{1} \preceq_{H} c_{2} \preceq_{H} \cdots \preceq_{H} c_{m} \preceq_{H} \cdots \preceq_{H} b\left(b \preceq_{H} \cdots \preceq_{H} c_{m} \preceq_{H} \cdots \preceq_{H} c_{2} \preceq_{H} c_{1}\right)$ for some $b \in L$, there exists $c \in L$ such that $\lim _{m \rightarrow \infty}\left\|c_{m}-c\right\|=0$.

Remark 2.5. Every regular cone is normal; see [51].

Definition 2.6. Let $L$ be an ordered normed space with normal solid cone $H$ and let $(X, P)$ be a Hausdorff cone uniform space with cone $H$.

(i) The family $\partial=\left\{J_{\alpha}: X \times X \rightarrow L, \alpha \in \mathcal{A}\right\}$ is said to be a 2 -family of cone pseudodistances on $X$ (2-family on $X$, for short) if the following three conditions hold:

(21) $\forall_{\alpha \in \mathcal{A}} \forall_{x, y \in X}\left\{0 \leq_{H} J_{\alpha}(x, y)\right\}$;

(22) $\forall_{\alpha \in \mathcal{A}} \forall_{x, y, z \in X}\left\{J_{\alpha}(x, z) \preceq_{H} J_{\alpha}(x, y)+J_{\alpha}(y, z)\right\}$;

(23) for any sequence $\left(w_{m}: m \in \mathbb{N}\right)$ in $X$ such that

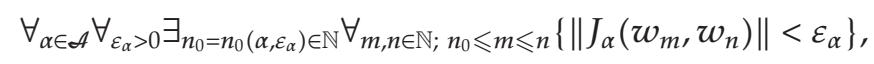

if there exists a sequence $\left(v_{m}: m \in \mathbb{N}\right)$ in $X$ satisfying

$$
\forall_{\alpha \in \mathcal{A}} \forall_{\mathcal{E}_{\alpha}>0} \exists_{n_{0}=n_{0}\left(\alpha, \varepsilon_{\alpha}\right) \in \mathbb{N}} \forall_{m \in \mathbb{N}} ; n_{0} \leqslant m\left\{\left\|J_{\alpha}\left(w_{m}, v_{m}\right)\right\|<\varepsilon_{\alpha}\right\},
$$

then

$$
\forall_{\alpha \in \mathcal{A}} \forall_{\mathcal{E}_{\alpha}>0} \exists_{n_{0}=n_{0}\left(\alpha, \varepsilon_{\alpha}\right) \in \mathbb{N}} \forall_{m \in \mathbb{N} ; n_{0} \leqslant m}\left\{\left\|p_{\alpha}\left(w_{m}, v_{m}\right)\right\|<\varepsilon_{\alpha}\right\}
$$

(ii) Let the family $\partial=\left\{J_{\alpha}: X \times X \rightarrow L, \alpha \in \mathcal{A}\right\}$ be a 2 -family on $X$. One says that a sequence $\left(w_{m}: m \in \mathbb{N}\right)$ in $X$ is a 2 -Cauchy sequence in $X$ if (2.5) holds.

Remark 2.7. Each $D$-family is a 2 -family.

The following result is useful.

Proposition 2.8. Let $(X, D)$ be a Hausdorff cone uniform space with cone $H$. Let the 2 -family $2=$ $\left\{J_{\alpha}: X \times X \rightarrow L, \alpha \in \mathcal{A}\right\}$ be a 2 -family. If $\forall_{\alpha \in \mathcal{A}}\left\{J_{\alpha}(x, y)=0 \wedge J_{\alpha}(y, x)=0\right\}$, then $x=y$.

Proof. Let $x, y \in X$ be such that $\forall_{\alpha \in \mathcal{A}}\left\{J_{\alpha}(x, y)=0 \wedge J_{\alpha}(y, x)=0\right\}$. By (22), $\forall_{\alpha \in \mathcal{A}}\left\{J_{\alpha}(x, x) \preceq_{H} J_{\alpha}(x, y)+J_{\alpha}(y, x)\right\}$. By (21), this gives $\forall_{\alpha \in \mathcal{A}}\left\{J_{\alpha}(x, x)=\right.$ $0\}$. Thus, we get $\forall_{\alpha \in \mathcal{A}} \forall_{\mathcal{E}_{\alpha}>0} \exists_{n_{0}=n_{0}\left(\alpha, \varepsilon_{\alpha}\right) \in \mathbb{N}} \forall_{m, n \in \mathbb{N} ; n_{0} \leqslant m \leqslant n}\left\{\left\|J_{\alpha}\left(w_{m}, w_{n}\right)\right\|<\varepsilon_{\alpha}\right\}$ and $\forall_{\alpha \in \mathscr{A}} \forall_{\varepsilon_{\alpha}>0} \exists_{n_{0}=n_{0}\left(\alpha, \varepsilon_{\alpha}\right) \in \mathbb{N}} \forall_{m \in \mathbb{N} ; n_{0} \leqslant m}\left\{\left\|J_{\alpha}\left(w_{m}, v_{m}\right)\right\|<\varepsilon_{\alpha}\right\}$ where $w_{m}=x, v_{m}=y$, and $m \in \mathbb{N}$, and, by (23), $\forall_{\alpha \in \mathcal{A}} \forall_{\varepsilon_{\alpha}>0} \exists_{n_{0}=n_{0}\left(\alpha, \varepsilon_{\alpha}\right) \in \mathbb{N}} \forall_{m \in \mathbb{N} ; n_{0} \leqslant m}\left\{\left\|p_{\alpha}\left(w_{m}, v_{m}\right)\right\|<\varepsilon_{\alpha}\right\}$, that is, $\forall_{\alpha \in \mathscr{A}} \forall_{\varepsilon_{\alpha}>0}\left\{\left\|p_{\alpha}(x, y)\right\|<\varepsilon_{\alpha}\right\}$. Hence, $\forall_{\alpha \in \mathcal{A}}\left\{p_{\alpha}(x, y)=0\right\}$ which, according to $(D 4)$, implies that $x=y$. 
Fixed Point Theory and Applications

\section{Maximality (Minimality) Principle in Cone Uniform Spaces with Generalized Pseudodistances}

We start with the following result.

Proposition 3.1. Let $L$ be an ordered Banach space with normal solid cone $H$, let $(X, D)$ be a Hausdorff cone uniform space with cone $H$ and let $\partial=\left\{J_{\alpha}: X \times X \rightarrow L, \alpha \in \mathcal{A}\right\}$ be ad-family on $X$. Every 2 -Cauchy sequence in $X$ is $P$-Cauchy sequence in $X$.

Proof. Indeed, assume that a sequence $\left(w_{m}: m \in \mathbb{N}\right)$ in $X$ is 2-Cauchy, that is, by Definition 2.6(ii), assume that

$$
\forall_{\alpha \in \mathscr{A}} \forall_{\mathcal{E}_{\alpha}>0} \exists_{n_{0}=n_{0}\left(\alpha, \varepsilon_{\alpha}\right) \in \mathbb{N}} \forall_{m, n \in \mathbb{N} ; n_{0} \leqslant m \leqslant n}\left\{\left\|J_{\alpha}\left(w_{m}, w_{n}\right)\right\|<\varepsilon_{\alpha}\right\}
$$

Hence $\forall_{\alpha \in \mathcal{A}} \forall_{\varepsilon_{\alpha}>0} \exists_{n_{0}=n_{0}\left(\alpha, \varepsilon_{\alpha}\right) \in \mathbb{N}} \forall_{m \in \mathbb{N}} ; n_{0} \leqslant m \forall_{q \in\{0\} \cup \mathbb{N}}\left\{\left\|J_{\alpha}\left(w_{m}, w_{q+m}\right)\right\|<\varepsilon_{\alpha}\right\}$, and if $i_{0} \in \mathbb{N}, j_{0} \in\{0\} \cup$ $\mathbb{N}, i_{0}>j_{0}$, and

$$
u_{m}=w_{i_{0}+m}, \quad v_{m}=w_{j_{0}+m} \text { for } m \in \mathbb{N},
$$

then

$$
\forall_{\alpha \in \mathscr{A}} \forall_{\varepsilon_{\alpha}>0} \exists_{n_{0}=n_{0}\left(\alpha, \varepsilon_{\alpha}\right) \in \mathbb{N}} \forall_{m \in \mathbb{N} ; n_{0} \leqslant m}\left\{\left\|J_{\alpha}\left(w_{m}, u_{m}\right)\right\|<\varepsilon_{\alpha} \wedge\left\|J_{\alpha}\left(w_{m}, v_{m}\right)\right\|<\varepsilon_{\alpha}\right\}
$$

By (23), (3.1) and (3.3),

$$
\forall_{\alpha \in \mathscr{A}} \forall_{\mathcal{E}_{\alpha}>0} \exists_{n_{0}=n_{0}\left(\alpha, \varepsilon_{\alpha}\right) \in \mathbb{N}} \forall_{m \in \mathbb{N} ; n_{0} \leqslant m}\left\{\left\|p_{\alpha}\left(w_{m}, u_{m}\right)\right\|<\varepsilon_{\alpha} \wedge\left\|p_{\alpha}\left(w_{m}, v_{m}\right)\right\|<\varepsilon_{\alpha}\right\} .
$$

If $M$ is a normal constant of $H$, then (3.2) and (3.4) give

$$
\forall_{\alpha \in \mathcal{A}} \forall_{\varepsilon_{\alpha}>0} \exists_{n_{0}=n_{0}\left(\alpha, \varepsilon_{\alpha}\right) \in \mathbb{N}} \forall_{m \in \mathbb{N} ; n_{0} \leqslant m}\left\{\left\|p_{\alpha}\left(w_{m}, w_{i_{0}+m}\right)\right\|<\frac{\varepsilon_{\alpha}}{2 M} \wedge\left\|p_{\alpha}\left(w_{m}, w_{j_{0}+m}\right)\right\|<\frac{\varepsilon_{\alpha}}{2 M}\right\} .
$$

Let $\alpha \in \mathcal{A}$ and $\varepsilon_{\alpha}>0$ be arbitrary and fixed and let $m, n \in \mathbb{N}$ satisfy $n_{0} \leqslant m<n$. We may suppose that $n=i_{0}+n_{0}$ and $m=j_{0}+n_{0}$ for some $i_{0} \in \mathbb{N}$ and $j_{0} \in\{0\} \cup \mathbb{N}$ such that $i_{0}>j_{0}$. Then, by $(D 1)-(D 3), \forall_{\alpha \in \mathcal{A}}\left\{0 \leq_{H} p_{\alpha}\left(w_{m}, \mathrm{w}_{n}\right)=p_{\alpha}\left(w_{j_{0}+n_{0}}, w_{i_{0}+n_{0}}\right) \leq_{H} p_{\alpha}\left(w_{n_{0}}, w_{j_{0}+n_{0}}\right)+p_{\alpha}\left(w_{n_{0}}, w_{i_{0}+n_{0}}\right)\right\}$. Hence, using (3.5), $\forall_{\alpha \in \mathcal{A}}\left\{\left\|p_{\alpha}\left(w_{m}, w_{n}\right)\right\| \leqslant M\left\|p_{\alpha}\left(w_{n_{0}}, w_{j_{0}+n_{0}}\right)\right\|+M\left\|p_{\alpha}\left(w_{n_{0}}, w_{i_{0}+n_{0}}\right)\right\|<\varepsilon_{\alpha}\right\}$ and, consequently, $\forall_{\alpha \in \mathcal{A}} \forall_{\mathcal{\varepsilon}_{\alpha}>0} \exists_{n_{0}=n_{0}\left(\alpha, \varepsilon_{\alpha}\right) \in \mathbb{N}} \forall_{m, n \in \mathbb{N} ; n_{0} \leqslant m<n}\left\{\left\|p_{\alpha}\left(w_{m}, w_{n}\right)\right\|<\varepsilon_{\alpha}\right\}$. Therefore, by Theorem 2.3(b), the sequence $\left(w_{m}: m \in \mathbb{N}\right)$ is $p$-Cauchy.

Let $\left(\Lambda, \leq_{\Lambda}\right)$ denote a directed set whose elements will be indicated by the letters $\lambda, \eta$, and $\mu$. In the sequel, $\lambda_{<_{\Lambda}} \eta$ will stand for $\lambda_{\leq_{\Lambda}} \eta$ and $\lambda \neq \eta$.

The relation $\leq_{X}$ on $X$ which is reflexive (i.e., for all $x \in X$ the condition $x \leq_{X} x$ holds) and transitive (i.e., for all $x, y, z \in X$ the conditions $x \leq_{X} y$ and $y \leq_{X} z$ imply that $x \leq_{X} z$ ) is called a quasiordering on $X$ and the pair $\left(X, \leq_{X}\right)$ is called a quasiordering space. If, additionally, relation $\leq_{\mathrm{X}}$ satisfies, for all $x, y \in X$, the conditions: $x \leq_{\mathrm{X}} y$ and $y \leq_{\mathrm{X}} x$ which imply that $x=y$, then it is called a partial quasiordering on $X$ and the pair $\left(X, \leq_{X}\right)$ is called a partial quasiordering space. In the sequel, $u<_{X} v$ will stand for $u \leq_{X} v$ and $u \neq v$. 
Definition 3.2. Let $L$ be an ordered normed space with solid cone $H$, let $(X, p)$ be a Hausdorff cone uniform space with cone $H$ and let $\partial=\left\{J_{\alpha}: X \times X \rightarrow L, \alpha \in \mathcal{A}\right\}$ be a $\partial$-family on $X$.

(i) One says that the net $\left(w_{\lambda}: \lambda \in \Lambda\right)$ in $X$ is 2-Cauchy ( $\not$ Cauchy) in $X$ if $\forall_{\alpha \in \mathcal{A}} \forall_{c_{\alpha} \in L, 0 \ll c_{\alpha}} \exists_{\pi_{0} \in \Lambda} \forall_{\eta, \mu \in \Lambda ; \pi_{0} \leq_{\Lambda} \eta \leq_{\Lambda} \mu}\left\{J_{\alpha}\left(w_{\eta}, w_{\mu}\right) \quad \ll \quad c_{\alpha}\right\}$

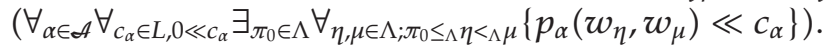

(ii) One says that the net ( $\left.w_{\lambda}: \lambda \in \Lambda\right)$ in $X$ is 2 -convergent ( $P$-convergent $)$ in $X$, if there exists $w \in X$ such that $\forall_{\alpha \in \mathcal{A}} \forall_{c_{\alpha} \in L, 0 \ll c_{\alpha}} \exists_{\pi_{0} \in \Lambda} \forall_{\eta \in \Lambda ; \pi_{0} \leq \Lambda}\left\{J_{\alpha}\left(w_{\eta}, w\right) \ll\right.$ $\left.c_{\alpha}\right\}\left(\forall_{\alpha \in \mathscr{A}} \forall_{c_{\alpha} \in L, 0 \ll c_{\alpha}} \exists_{\pi_{0} \in \Lambda} \forall_{\eta \in \Lambda ; \pi_{0} \leq \Lambda \eta}\left\{p_{\alpha}\left(w_{\eta}, w\right) \ll c_{\alpha}\right\}\right)$.

(iii) One says that $(X, D)$ is complete, if every $p$-Cauchy net $\left(w_{\lambda}: \lambda \in \Lambda\right)$ in $X$ is $p$ convergent in $X$.

(iv) Let $(X, D)$ be complete. For an arbitrary subset $E$ of $X$, the closure of $E$, denoted by $\mathrm{cl}(E)$, is defined as the set $\mathrm{cl}(E)=\{w \in X$ : $\left.\exists_{\left(w_{i}: \lambda \in \Lambda\right) \subset E} \forall_{\alpha \in \mathcal{A}} \forall_{c_{\alpha} \in L, 0 \ll c_{\alpha}} \exists_{\pi_{0} \in \Lambda} \forall_{\eta \in \Lambda ; \pi_{0} \leq \Lambda}\left\{p_{\alpha}\left(w_{\eta}, w\right) \ll c_{\alpha}\right\}\right\}$. The subset $E$ of $X$ is said to be a closed subset in $X$ if $\operatorname{cl}(E)=E$.

(v) Let $\left(X, \leq_{X}\right)$ be a partial quasiordering space. One says that the net $\left(w_{\lambda}: \lambda \in\right.$ $\Lambda)$ in $\left(X, \leq_{X}\right)$ is increasing (decreasing) with respect to $\leq_{X}$ if $\forall_{\eta, \mu \in \Lambda}\left\{\eta<_{\Lambda} \mu \Rightarrow\right.$ $\left.w_{\eta} \leq_{X} w_{\mu}\right\}\left(\forall_{\eta, \mu \in \Lambda}\left\{\eta<_{\Lambda} \mu \Rightarrow w_{\mu} \leq_{X} w_{\eta}\right\}\right)$.

Of course, each $p$-convergent net is a $p$-Cauchy net. Also we show the following

Proposition 3.3. Let $L$ be an ordered Banach space with a solid cone $H$ and let $(X, D)$ be a Hausdorff cone uniform space with cone $H$. Let $\partial=\left\{J_{\alpha}: X \times X \rightarrow L, \alpha \in \mathcal{A}\right\}$ be a -family on $X$ and let $\left(X, \leq_{X}\right)$ be a partial quasiordering space.

(a) Assume that each increasing sequence $\left(w_{m}: m \in \mathbb{N}\right)$ in $X$ is 2 -Cauchy ( $D$-Cauchy). Then each increasing net $\left(w_{\lambda}: \lambda \in \Lambda\right)$ in $X$ is 2 -Cauchy ( $D$-Cauchy).

(b) Assume that each decreasing sequence $\left(w_{m}: m \in \mathbb{N}\right)$ in $X$ is 2 -Cauchy ( $P$-Cauchy). Then each decreasing net ( $\left.w_{\lambda}: \lambda \in \Lambda\right)$ in $X$ is 2 -Cauchy ( $D$-Cauchy).

Proof. (a) Suppose that there exists an increasing net $\left(w_{\lambda}: \lambda \in \Lambda\right)$ in $X$ which is not 2-Cauchy, that is, which satisfies $\forall_{\eta, \mu \in \Lambda}\left\{\eta<_{\Lambda} \mu \Rightarrow w_{\eta} \leq_{\mathrm{X}} w_{\mu}\right\}$ and

$$
\exists_{\alpha_{0} \in \mathcal{A}} \exists_{c_{\alpha_{0}} \in L, 0 \ll c_{\alpha_{0}}} \forall_{\pi \in \Lambda} \exists_{\eta, \mu \in \Lambda ; \pi \leq \Lambda} \eta \leq_{\Lambda} \mu\left\{J_{\alpha_{0}}\left(w_{\eta}, w_{\mu}\right)-c_{\alpha_{0}} \notin \operatorname{int}(H)\right\} .
$$

Assume that $\pi_{1} \in \Lambda$ is arbitrary and fixed. By (3.6), there exist $\eta_{1}, \mu_{1} \in \Lambda$, $\pi_{1} \leq_{\Lambda} \eta_{1} \leq \Lambda \mu_{1}$, such that $J_{\alpha_{0}}\left(w_{\eta_{1}}, w_{\mu_{1}}\right)-c_{\alpha_{0}} \notin \operatorname{int}(H)$ and define $v_{1}=w_{\eta_{1}}$ and $v_{2}=w_{\mu_{1}}$. Next, for $\pi_{2}=\mu_{1}$, by (3.6), there exist $\eta_{2}, \mu_{2} \in \Lambda, \pi_{2} \leq_{\Lambda} \eta_{2} \leq_{\Lambda} \mu_{2}$, such that $J_{\alpha_{0}}\left(w_{\eta_{2}}, w_{\mu_{2}}\right)-c_{\alpha_{0}} \notin \operatorname{int}(H)$ and define $v_{3}=w_{\eta_{2}}$ and $v_{4}=w_{\mu_{2}}$. Now, if $v_{k}$ are defined for $k=1, \ldots, 2(n-1)$ and if $\pi_{n}=\mu_{n-1}$, then, by (3.6), there exist $\eta_{n}, \mu_{n} \in \Lambda, \pi_{n} \leq_{\Lambda} \eta_{n} \leq_{\Lambda} \mu_{n}$, such that $J_{\alpha_{0}}\left(w_{\eta_{n}}, w_{\mu_{n}}\right)-c_{\alpha_{0}} \notin \operatorname{int}(H)$ and define $v_{2 n-1}=w_{\eta_{n}}$ and $v_{2 n}=w_{\mu_{n}}$. By induction, this gives $\forall_{m \in \mathbb{N}}\left\{v_{m} \leq_{X} v_{m+1}\right\}$ and $\exists_{\alpha_{0} \in \mathcal{A}} \exists_{\mathcal{c}_{\alpha_{0}} \in L, 0<c_{\alpha_{0}}} \forall_{n \in \mathbb{N}} \exists_{m_{0}, n_{0} \in \mathbb{N} ; n \leqslant m_{0} \leqslant n_{0}}\left\{J_{\alpha_{0}}\left(v_{m_{0}}, v_{n_{0}}\right)-c_{\alpha_{0}} \notin \operatorname{int}(H)\right\}$. Consequently, there exists an increasing sequence $\left(v_{n}: n \in \mathbb{N}\right)$ in $X$ which is not 2-Cauchy.

By Remark 2.7, we get the claim.

(b) We use a similar argument as in (a). 
Let $\left(X, \leq_{X}\right)$ be a partial quasiordering space. Set $E \subset X$ which is called a chain in $X$ if any two elements of $E$ are comparable, that is, $x \leq_{X} y$ or $y \leq_{X} x$ for all $x, y \in E$. The Zorn lemma says that every partially ordered set in which every chain has an upper (lower) bound contains at least one maximal (minimal) element.

The main result of this section is the following maximality (minimality) principle.

Theorem 3.4. Let $L$ be an ordered Banach space with a normal solid cone $H$ and let $(X, D)$ be a Hausdorff cone uniform space with cone $H$. Let $\partial=\left\{J_{\alpha}: X \times X \rightarrow L, \alpha \in \mathcal{A}\right\}$ be ad-family on $X$ and let $\left(X, \leq_{X}\right)$ be a partial quasiordering space.

(A) Assume that $\left(a_{1}\right)$ for each $x \in X$, the set $\left\{y \in X: x \leq_{X} y\right\}$ is complete, and $\left(a_{2}\right)$ each increasing sequence $\left(w_{m}: m \in \mathbb{N}\right)$ in $X$ is 2 -Cauchy. Then $X$ contains at least one maximal element.

(B) Assume that $\left(b_{1}\right)$ for each $x \in X$, the set $\left\{y \in X: y \leq_{X} x\right\}$ is complete, and $\left(b_{2}\right)$ each decreasing sequence $\left(w_{m}: m \in \mathbb{N}\right)$ in $X$ is 2 -Cauchy. Then $X$ contains at least one minimal element.

Proof. (A) The proof will be broken into five steps.

Step 1. Suppose $\left(\mathrm{a}_{2}\right)$ holds, that is, that each increasing sequence $\left(w_{m}: m \in \mathbb{N}\right)$ in $X$ is 2 Cauchy. Then, by Proposition 3.1, each increasing sequence $\left(w_{m}: m \in \mathbb{N}\right)$ in $X$ is $D$-Cauchy and, consequently, Proposition 3.3(a) gives that each increasing net $\left(w_{\lambda}: \lambda \in \Lambda\right)$ in $X$ is p-Cauchy.

Step 2. Let an increasing net $\left(w_{\lambda}: \lambda \in \Lambda\right)$ in $X$ be arbitrary and fixed. In view of $\left(\mathrm{a}_{1}\right)$ and Step $1,\left(w_{\curlywedge}: \lambda \in \Lambda\right)$ is convergent to a $w \in X$ and, since $X$ is Hausdorff, $w$ is unique.

Step 3. Let $E$ be a chain in $\left(X, \leq_{X}\right)$. If $\exists_{u \in E} \forall_{v \in E}\left\{v \leq_{X} u\right\}$, then $E$ has an upper bound in $X$.

Step 4. Let $E$ be a chain in $\left(X, \leq_{X}\right)$. If $\forall_{u \in E} \exists_{v \in E}\left\{u<_{X} v\right\}$, then denoting $\Lambda=E$ and $w_{\lambda}=\lambda$ for each $\lambda \in E$, we can identify $E$ with the increasing net $\left(w_{\lambda}: \lambda \in \Lambda\right)$. Next, using, in particular, Steps 1 and 2, we can show that $\forall_{\lambda \in \Lambda}\left\{w_{\lambda} \leq_{X} w\right\}$ where $w$ is a unique limit of $\left(w_{\lambda}: \lambda \in \Lambda\right)$; which means that $w$ is an upper bound of $\left(w_{\lambda}: \lambda \in \Lambda\right)$. Indeed, let $\lambda_{0} \in \Lambda$ be arbitrary and fixed and define the sets $\Lambda_{0}, E_{0}$ by $\Lambda_{0}=\left\{\lambda \in \Lambda: \lambda_{0} \leq_{\Lambda} \lambda\right\}, E_{0}=\left\{y \in X: w_{\lambda_{0}} \leq x y\right\}$. By assumption $\left(\mathrm{a}_{1}\right), E_{0}$ is complete. Clearly, the net $\left(w_{\lambda}: \lambda \in \Lambda_{0}\right)$ is increasing in $X, P$-Cauchy, convergent to $w$ and $w \in E_{0}$. This proves that $w_{\lambda_{0}} \leq \mathrm{X} w$. Therefore, $E$ has an upper bound in $X$.

Step 5. Using Steps 3 and 4 and the Zorn lemma, we conclude that $X$ contains at least one maximal element.

(B) We use a similar argument as in (A).

\section{Variational Principle of Ekeland Type and Fixed Point and Endpoint Theorem of Caristi Type in Cone Uniform Spaces with Generalized Pseudodistances}

Let $2^{X}$ denote the family of all nonempty subsets of a space $X$. Recall that a set-valued dynamic system is defined as a pair $(X, T)$, where $X$ is a certain space and $T$ is a set-valued map 
$T: X \rightarrow 2^{X}$; in particular, a set-valued dynamic system includes the usual dynamic system where $T$ is a single-valued map.

Let $L$ be an ordered Banach space with a cone $H$ and let $(X, D)$ be a cone uniform space with cone $H$.

Let an element $+\infty \notin L$ be such that $a \leq_{H}+\infty$ for all $a \in L$. We say that a map $F: X \rightarrow$ $L \cup\{+\infty\}$ is proper if its effective domain, $\operatorname{dom}(F)=\{x: \omega(x) \neq+\infty\}$, is nonempty.

If $\mathcal{\partial}=\left\{J_{\alpha}: X \times X \rightarrow L: \alpha \in \mathcal{A}\right\}$ is a 2 -family, then

$$
X=X_{2}^{0} \cup X_{2}^{+}
$$

where

$$
\begin{aligned}
& X_{\partial}^{0}=\left\{x \in X: \forall_{\alpha \in \mathcal{A}}\left\{0=J_{\alpha}(x, x)\right\}\right\}, \\
& X_{\partial}^{+}=\left\{x \in X: \exists_{\alpha \in \mathcal{A}}\left\{0 \prec_{H} J_{\alpha}(x, x)\right\}\right\} .
\end{aligned}
$$
type.

Using Theorem 3.4(B), we can prove the following variational principle of Ekeland

Theorem 4.1. Assume that

(a) $L$ is an ordered Banach space with a regular solid cone $H$;

(b) $(X, D)$ is a Hausdorff complete cone uniform space with cone $H$;

(c) the family $\partial=\left\{J_{\alpha}: X \times X \rightarrow L, \alpha \in \mathcal{A}\right\}$ is a 2 -family on $X$ such that $X_{\partial}^{0} \neq \emptyset$;

(d) the family $\Omega=\left\{\omega_{\alpha}: X \rightarrow H \cup\{+\infty\}, \alpha \in \mathscr{A}\right\}$ satisfies $D_{\Omega}=\bigcap_{\alpha \in \mathcal{A}} \operatorname{dom}\left(\omega_{\alpha}\right) \neq \emptyset$;

(e) $\left\{\varepsilon_{\alpha}, \alpha \in \mathcal{A}\right\}$ is a family of finite positive numbers;

(f) for each $x \in X_{2}^{0}$, the set $Q_{2, \Omega}(x)$ defined by the formula

$$
Q_{\partial, \Omega}(x)=\left\{y \in X_{\partial}^{0}: \forall_{\alpha \in \mathcal{A}}\left\{\omega_{\alpha}(y)+\varepsilon_{\alpha} J_{\alpha}(x, y) \preceq_{H} \omega_{\alpha}(x)\right\}\right\}
$$

is a nonempty closed subset in $X$.

Then, for each $w_{0} \in D_{\Omega} \cap X_{2}^{0}$, there exists $w \in D_{\Omega} \cap X_{2}^{0}$ such that

(i) $\forall_{\alpha \in \mathcal{A}}\left\{\omega_{\alpha}(w)+\varepsilon_{\alpha} J_{\alpha}\left(w_{0}, w\right) \varliminf_{H} \omega_{\alpha}\left(w_{0}\right)\right\}$;

(ii) $\forall_{x \in Q_{2, \Omega}\left(w_{0}\right) \backslash\{w\}} \exists_{\beta \in \mathcal{A}}\left\{\omega_{\beta}(w) \prec_{H} \omega_{\beta}(x)+\varepsilon_{\beta} J_{\beta}(x, w)\right\}$;

(iii) if $w \neq w_{0}$, then $\exists_{\gamma \in \mathcal{A}}\left\{\omega_{\gamma}(w) \prec_{H} \omega_{\gamma}\left(w_{0}\right)\right\}$.

Proof. The key observation in the proof is that $\left(X, Z_{2, \Omega}\right)$ is a set-valued dynamic system where

$$
Z_{2, \Omega}(x)= \begin{cases}Q_{2, \Omega}(x) & \text { if } x \in X_{2}^{0} \\ \{x\} & \text { if } x \in X_{2}^{+}\end{cases}
$$

and, by assumption (f), for each $x \in X, Z_{2, \Omega}(x)$ is a closed subset in $X$.

The proof will be broken into five steps. 
Fixed Point Theory and Applications

Step 1. The following shrinking property holds:

$$
\forall_{w_{0} \in X} \forall_{v \in Z_{2, \Omega}\left(w_{0}\right)}\left\{Z_{2, \Omega}(v) \subset Z_{2, \Omega}\left(w_{0}\right)\right\}
$$

Let $w_{0} \in X, v \in Z_{2, \Omega}\left(w_{0}\right)$ and $u \in Z_{2, \Omega}(v)$ be arbitrary and fixed. Then $u \in Z_{2, \Omega}\left(w_{0}\right)$. Indeed, we have the following.

Case 1. Assuming that $w_{0} \in X_{2}^{0}$, we get $v \in Z_{2, \Omega}\left(w_{0}\right)=Q_{2, \Omega}\left(w_{0}\right)$. Hence, by definition of $Q_{2, \Omega}\left(w_{0}\right), v \in X_{2}^{0}$. Consequently, $u \in Z_{2, \Omega}(v)$ implies that $u \in Q_{2, \Omega}(v)$ and, by (22), we obtain $\forall_{\alpha \in \mathcal{A}}\left\{\omega_{\alpha}(u)+\varepsilon_{\alpha} J_{\alpha}\left(w_{0}, u\right) \leq_{H} \omega_{\alpha}(u)+\varepsilon_{\alpha} J_{\alpha}(v, u)+\varepsilon_{\alpha} J_{\alpha}\left(w_{0}, v\right) \leq_{H} \omega_{\alpha}(v)+\right.$ $\left.\varepsilon_{\alpha} J_{\alpha}\left(w_{0}, v\right) \preceq_{H} w_{\alpha}\left(w_{0}\right)\right\}$, that is, $u \in Q_{2, \Omega}\left(w_{0}\right)=Z_{2, \Omega}\left(w_{0}\right)$, which gives (4.5).

Case 2. Assuming that $w_{0} \in X_{2}^{+}$, we get $v \in Z_{2, \Omega}\left(w_{0}\right)=\left\{w_{0}\right\}$. Hence $v=u=w_{0}$. This gives (4.5).

Step 2. Let $w_{0} \in X$ be arbitrary and fixed. Define the relation $\leq_{Z_{2, \Omega}\left(w_{0}\right)}$ on $Z_{2, \Omega}\left(w_{0}\right)$ as follows:

$$
\forall_{u_{1}, u_{2} \in Z_{2, \Omega}\left(w_{0}\right)}\left\{u_{2} \leq_{2, \Omega}\left(w_{0}\right) u_{1} \Longleftrightarrow u_{2}=u_{1} \text {, if } u_{1} \in X_{2}^{+} \text {, or } u_{2} \in Q_{2, \Omega}\left(u_{1}\right), \text { if } u_{1} \in X_{2}^{0}\right\} .
$$

Then $\left(Z_{2, \Omega}\left(w_{0}\right), \leq_{2, \Omega}\left(w_{0}\right)\right)$ is a partial quasiordering space.

Remark 4.2. It is worth noticing that, for $w_{0} \in X$ and $u_{1}, u_{2} \in Z_{2, \Omega}\left(w_{0}\right)$, if $w_{0} \in X_{2}^{0}$, then $u_{1}, u_{2} \in X_{2}^{0}$ and if $w_{0} \in X_{2}^{+}$, then $u_{1}=u_{2}=w_{0} \in X_{2}^{+}$.

Relation $\leq_{Z_{2, \Omega}\left(w_{0}\right)}$ on $Z_{2, \Omega}\left(w_{0}\right)$ is reflexive. We show that, for each $u \in Z_{2, \Omega}\left(w_{0}\right)$, $u \leq_{Z_{2, \Omega}\left(w_{0}\right)} u$. Indeed, assuming that $w_{0} \in X_{2}^{0}$ we have $u \in X_{2}^{0}$ and, consequently, we have $\forall_{\alpha \in \mathcal{A}}\left\{0=J_{\alpha}(u, u)\right\}$ which gives $u \in Q_{2, \Omega}(u)$ and thus $u \leq_{Z_{2, \Omega}\left(w_{0}\right)} u$. Assuming that $w_{0} \in X_{2}^{+}$we have that $u=w_{0} \in X_{2}^{+}$and thus we get that $u \leq z_{2, \Omega}\left(w_{0}\right) u$.

Relation $\leq_{z_{2, \Omega}\left(w_{0}\right)}$ on $Z_{2, \Omega}\left(w_{0}\right)$ is transitive. Indeed, let $u \leq_{z_{2, \Omega}\left(w_{0}\right)} v$ and $v \leq_{z_{2, \Omega}\left(w_{0}\right)} z$ for $u, v, z \in Z_{2, \Omega}\left(w_{0}\right)$. Clearly, by Remark 4.2 , we have that $u, v, z \in X_{2}^{0}$ if $w_{0} \in X_{2}^{0}$ or $u, v, z \in X_{2}^{+}$ if $w_{0} \in X_{2}^{+}$. If $v, z \in X_{2}^{0}$, then $u \in Q_{2, \Omega}(v)$ and $v \in Q_{2, \Omega}(z)$. Hence, using (22), we obtain $\forall_{\alpha \in \mathcal{A}}\left\{\omega_{\alpha}(u)+\varepsilon_{\alpha} J_{\alpha}(z, u) \leq_{H} \omega_{\alpha}(u)+\varepsilon_{\alpha}\left(J_{\alpha}(z, v)+J_{\alpha}(v, u)\right) \leq_{H} \omega_{\alpha}(z)\right\}$ which gives $u \leq_{Z_{2, \Omega}\left(w_{0}\right)} z$. If $v, z \in X_{2}^{+}$, then $u=v=z$ which implies that $u \leq_{2, \Omega}\left(w_{0}\right) z$.

Relation $\leq_{Z_{2, \Omega}\left(w_{0}\right)}$ on $Z_{2, \Omega}\left(w_{0}\right)$ is partial. Indeed, let $u \leq_{Z_{2, \Omega}\left(w_{0}\right)} v$ and $v \leq_{Z_{2, \Omega}\left(w_{0}\right)} u$ for $u, v \in Z_{2, \Omega}\left(w_{0}\right)$. Then, by Remark $4.2, u, v \in X_{2}^{0}$ if $w_{0} \in X_{2}^{0}$ or $u, v \in X_{2}^{+}$if $w_{0} \in X_{2}^{+}$. If $v, u \in X_{2}^{0}$, then $u \in Q_{2, \Omega}(v)$ and $v \in Q_{2, \Omega}(u)$. By order $\varsigma_{H}$, we conclude that the conditions $v \in Q_{\partial, \Omega}(u), \forall_{\alpha \in \mathcal{A}}\left\{\omega_{\alpha}(u)-\left[\omega_{\alpha}(v)+\varepsilon_{\alpha} J_{\alpha}(u, v)\right]=\left[\omega_{\alpha}(u)-\varepsilon_{\alpha} J_{\alpha}(u, v)\right]-\omega_{\alpha}(v)\right\} \in H$ and

$$
\forall_{\alpha \in \mathcal{A}}\left\{\omega_{\alpha}(v) \leq_{H} \omega_{\alpha}(u)-\varepsilon_{\alpha} J_{\alpha}(u, v)\right\}
$$

are equivalent. Further, $u \in Q_{2, \Omega}(v)$ means that

$$
\forall_{\alpha \in \mathcal{A}}\left\{\omega_{\alpha}(u)+\varepsilon_{\alpha} J_{\alpha}(v, u) \leq_{H} \omega_{\alpha}(v)\right\}
$$


In virtue of (4.8), (4.7) and transitive property of order $\preceq_{H}$, we obtain that

$$
\forall_{\alpha \in \mathcal{A}}\left\{\omega_{\alpha}(u)+\varepsilon_{\alpha} J_{\alpha}(v, u) \varliminf_{H} \omega_{\alpha}(u)-\varepsilon_{\alpha} J_{\alpha}(u, v)\right\}
$$

In conclusion, $\forall_{\alpha \in \mathcal{A}}\left\{-\varepsilon_{\alpha}\left[J_{\alpha}(u, v)+J_{\alpha}(v, u)\right] \in H\right\}$. Hence, by $(21), \forall_{\alpha \in \mathcal{A}}\left\{J_{\alpha}(u, v)=0 \wedge\right.$ $\left.J_{\alpha}(v, u)=0\right\}$. Therefore, Proposition 2.8 implies that $u=v$.

If $v, u \in X_{2}^{+}$, then $u=v$.

Step 3. Let $w_{0} \in X$ be arbitrary and fixed. For each $v \in Z_{2, \Omega}\left(w_{0}\right)$, the set $\left\{u \in Z_{2, \Omega}\left(w_{0}\right)\right.$ : $\left.u \leq z_{2, \Omega}\left(w_{0}\right) v\right\}$ is complete.

Case 1. Assume that $w_{0} \in X_{2}^{0}$. Therefore $Z_{2, \Omega}\left(w_{0}\right)=Q_{2, \Omega}\left(w_{0}\right) \subset X_{2}^{0}$. However, $v \in$ $Z_{2, \Omega}\left(w_{0}\right) \subset X_{2}^{0}$. Hence, by Step 1 and (4.6), we obtain that $\left\{u \in Z_{2, \Omega}\left(w_{0}\right): u \leq_{Z_{2, \Omega}\left(w_{0}\right)} v\right\}=$ $Z_{2, \Omega}(v)=Q_{2, \Omega}(v) \subset X_{2}^{0}$ which, by (f), (b) and Definition 3.2(iv), implies that the set $\left\{u \in Z_{2, \Omega}\left(w_{0}\right): u \leq_{Z_{2, \Omega}\left(w_{0}\right)} v\right\}$ is complete.

Case 2. Assume that $w_{0} \in X_{2}^{+}$. Then $Z_{2, \Omega}\left(w_{0}\right)=\left\{w_{0}\right\}$ and the set $\left\{u \in Z_{2, \Omega}\left(w_{0}\right)\right.$ : $\left.u \leq_{Z_{2, \Omega}\left(w_{0}\right)} v\right\}=\left\{w_{0}\right\}$ is complete.

Step 4. Let $w_{0} \in X$ be arbitrary and fixed. Each decreasing (with respect to $\left.\leq_{Z_{2, \Omega}\left(w_{0}\right)}\right)$ sequence $\left(w_{m}\right.$ : $m \in \mathbb{N})$ in $Z_{2, \Omega}\left(w_{0}\right)$ is 2 -Cauchy.

Indeed, let $\left(w_{m}: m \in \mathbb{N}\right)$ be a decreasing sequence in $Z_{2, \Omega}\left(w_{0}\right)$, that is, $\forall_{m \in \mathbb{N}}\left\{w_{m+1} \leq_{Z_{2, \Omega}\left(w_{0}\right)} w_{m}\right.$ and $\left.w_{m} \in Z_{2, \Omega}\left(w_{0}\right)\right\}$.

Case 1. If $w_{0} \in X_{2}^{0}$, then, for each $m \in \mathbb{N}, w_{m} \in X_{2}^{0}$. Therefore, $\forall_{m \in \mathbb{N}}\left\{w_{m+1} \in\right.$ $\left.Q_{2, \Omega}\left(w_{m}\right)\right\}$, that is, $\forall_{m \in \mathbb{N}} \forall_{\alpha \in \mathcal{A}}\left\{w_{\alpha}\left(w_{m+1}\right)+\varepsilon_{\alpha} J_{\alpha}\left(w_{m}, w_{m+1}\right) \leq_{H} w_{\alpha}\left(w_{m}\right)\right\}$. Hence, by (d), $\forall_{m \in \mathbb{N}} \forall_{\alpha \in \mathcal{A}}\left\{0 \leq_{H} \cdots \preceq_{H} \omega_{\alpha}\left(w_{m+1}\right) \leq_{H} \omega_{\alpha}\left(w_{m}\right) \leq_{H} \cdots\right\}$ and since $H$ is a closed and regular cone, it follows that

$$
\forall_{\alpha \in \mathcal{A}} \exists_{\mathfrak{u}_{\alpha} \in H}\left\{\lim _{m \rightarrow \infty}\left\|\omega_{\alpha}\left(w_{m}\right)-u_{\alpha}\right\|=0\right\} .
$$

Moreover,

$$
\forall_{m \in \mathbb{N}} \forall_{\alpha \in \mathcal{A}}\left\{0 \preceq_{H} u_{\alpha} \preceq_{H} \cdots \preceq_{H} \omega_{\alpha}\left(w_{m+1}\right) \preceq_{H} \omega_{\alpha}\left(w_{m}\right) \preceq_{H} \cdots\right\}
$$

Indeed, let $m_{0} \in \mathbb{N}$ and $\alpha_{0} \in \mathcal{A}$ be arbitrary and fixed. Then, $\forall_{n \in \mathbb{N}}\left\{\omega_{\alpha_{0}}\left(w_{m_{0}}\right)-\omega_{\alpha_{0}}\left(w_{m_{0}+n}\right) \in H\right\}$. Consequently, since $H$ is closed, by (4.10), $\lim _{n}\left\{\omega_{\alpha_{0}}\left(w_{m_{0}}\right)-\omega_{\alpha_{0}}\left(w_{m_{0}+n}\right)\right\}=\omega_{\alpha_{0}}\left(w_{m_{0}}\right)-u_{\alpha_{0}} \in H$. This gives (4.11).

On the other hand, if $m \leqslant n$, then, in virtue of (21) and (22), we derive $\forall_{\alpha \in \mathcal{A}}\left\{0 \leq_{H} \varepsilon_{\alpha} J_{\alpha}\left(w_{m}, w_{n}\right) \varliminf_{H} \sum_{j=m}^{n-1} \varepsilon_{\alpha \alpha} J_{\alpha}\left(w_{j}, w_{j+1}\right) \varliminf_{H} w_{\alpha}\left(w_{m}\right) \quad-\quad u_{\alpha} \quad-\right.$ $\left.\left(\omega_{\alpha}\left(w_{n}\right)-u_{\alpha}\right)\right\}$. From this, since $H$ is normal (see Remark 2.5), we conclude that $\forall_{\alpha \in \mathcal{A}} \forall_{\eta_{\alpha}>0} \exists_{n_{0} \in \mathbb{N}} \forall \forall_{m, n \in \mathbb{N} ; n_{0} \leqslant m \leqslant n}\left\{\left\|J_{\alpha}\left(w_{m}, w_{n}\right)\right\|<\eta_{\alpha}\right\}$. Therefore, by Definition 2.6(ii), Proposition 3.1 and Theorem 2.3(b), $\left(w_{m}: m \in \mathbb{N}\right)$ is $p$-Cauchy.

Case 2. If $w_{0} \in X_{2}^{+}$, then, for each $m \in \mathbb{N}, w_{m}=w_{0}$ and $\left(w_{m}: m \in \mathbb{N}\right)$ is $p$-Cauchy. 
Step 5. Let $w_{0} \in D_{\Omega} \cap X_{2}^{0}$ be arbitrary and fixed. Then there exists $w \in Q_{2, \Omega}\left(w_{0}\right)$ such that $\{w\}=$ $Q_{2, \Omega}(w)$.

Since $w_{0} \in D_{\Omega} \cap X_{2}^{0}$, thus $Z_{2, \Omega}\left(w_{0}\right)=Q_{2, \Omega}\left(w_{0}\right) \neq \emptyset$ and, by Steps $1-4$ and Theorem 3.4(B), $Z_{2, \Omega}\left(w_{0}\right)$ has a minimal element $w$. Of course, $w \in Q_{2, \Omega}\left(w_{0}\right)$ gives (i). Moreover, denoting that $V=\left\{v \in Q_{2, \Omega}\left(w_{0}\right): v \leq_{Z_{2, \Omega}\left(w_{0}\right)} w\right\}$ we conclude that $V=\{v \in$ $\left.Q_{2, \Omega}\left(w_{0}\right): v \in Q_{2, \Omega}(w)\right\}=\{w\}$. Therefore, $w$ is an endpoint of $Q_{2, \Omega}$ in $Q_{2, \Omega}\left(w_{0}\right)$, that is, $\{w\}=Q_{2, \Omega}(w)$; we see, by $(21)$, that $\{w\}=Q_{2, \Omega}(w)$ gives $\forall_{\alpha \in \mathcal{A}}\left\{J_{\alpha}(w, w)=0\right\}$, that is, $w \in X_{2}^{0}$. Of course, $\{w\}=Q_{2, \Omega}(w)$ implies that $\forall_{x \in Q_{2, \Omega}\left(w_{0}\right) \backslash\{w\}}\left\{x \notin Q_{2, \Omega}(w)\right\}$, that is, (ii) holds. Assertion (iii) follows from (i) and (ii).

Definition 4.3. Let $(X, D)$ be a Hausdorff complete cone uniform space with cone $H$. Let $E \subseteq X$, $E \neq \emptyset$. The map $F: E \rightarrow H \cup\{+\infty\}$ is lower semicontinuous on $E$ with respect to $X$ (written: $F$ is $(E, X)$-lsc when $E \neq X$ and $F$ is $l s c$ when $E=X)$ if the set $\left\{y \in E: F(y) \preceq_{H} c\right\}$ is closed subset in $X$ for each $c \in H$.

Remark 4.4. (a) A special case of condition (f) is a condition $\left(\mathrm{f}^{\prime}\right)$ defined by

$\left(\mathrm{f}^{\prime}\right)$ for each $(x, \alpha) \in X_{2}^{0} \times \mathcal{A}$, the map $\omega_{\alpha}(\cdot)+\varepsilon_{\alpha} J_{\alpha}(x, \cdot): X_{2}^{0} \rightarrow H \cup\{+\infty\}$ is $\left(X_{2}^{0}, X\right)$-lsc and, for each $x \in X_{2}^{0}$, the set $Q_{2, \Omega}(x)$ is nonempty.

(b) If $\partial=p$, then a special case of condition ( $\mathrm{f}$ ) is a condition ( $\left.\mathrm{f}^{\prime \prime}\right)$ defined by

$\left(\mathrm{f}^{\prime \prime}\right)$ for each $(x, \alpha) \in X \times \mathcal{A}$, the map $\omega_{\alpha}(\cdot)+\varepsilon_{\alpha} p_{\alpha}(x, \cdot): X \rightarrow H \cup\{+\infty\}$ is lsc and, for each $x \in X$, the set $Q_{p, \Omega}(x)$ is nonempty.

Let $(X, T)$ be a set-valued dynamic system. By $\operatorname{Fix}(T)$ and $\operatorname{End}(T)$ we denote the sets of all fixed points and endpoints of $T$, respectively, that is, $\operatorname{Fix}(T)=\{w \in X: w \in T(w)\}$ and $\operatorname{End}(T)=\{w \in X:\{w\}=T(w)\}$.

A dynamic process or a trajectory starting at $w_{0} \in X$ or a motion of the system $(X, T)$ at $w_{0}$ is a sequence $\left(w_{m}: m \in\{0\} \cup \mathbb{N}\right.$ ) defined by $w_{m} \in T\left(w_{m-1}\right)$ for $m \in \mathbb{N}$ (see,Aubin and Siegel [4], and Yuan [52]).

The following fixed point and endpoint theorem of Caristi type holds.

Theorem 4.5. Assume that

(a) $L$ is an ordered Banach space with a regular solid cone $H$;

(b) $(X, D)$ is a Hausdorff complete cone uniform space with cone $H$;

(c) the family $\partial=\left\{J_{\alpha}: X \times X \rightarrow L, \alpha \in \mathcal{A}\right\}$ is a $\partial$-family on $X$ such that $X_{\partial}^{0} \neq \emptyset$;

(d) the family $\Omega=\left\{\omega_{\alpha}: X \rightarrow H \cup\{+\infty\}, \alpha \in \mathcal{A}\right\}$ satisfies $D_{\Omega}=\bigcap_{\alpha \in \mathscr{A}} \operatorname{dom}\left(\omega_{\alpha}\right) \neq \emptyset$;

(e) $\left\{\varepsilon_{\alpha}, \alpha \in \mathcal{A}\right\}$ is a family of finite positive numbers;

(f) $(X, T)$ is a set-valued dynamic system;

(g) for each $x \in X_{2}^{0}$, the set $Q_{2, \Omega ; T}(x)$ defined by the formula

$$
Q_{\partial, \Omega ; T}(x)=\left\{y \in T(x) \cap X_{\partial}^{0}: \forall_{\alpha \in \mathcal{A}}\left\{\omega_{\alpha}(y)+\varepsilon_{\alpha} J_{\alpha}(x, y) \preceq_{H} \omega_{\alpha}(x)\right\}\right\}
$$

is a nonempty closed subset in X. 
Then, there exists $w \in D_{\Omega} \cap X_{2}^{0}$ such that

(i) $w \in T(w)$.

Assume, in addition, that

(h) for each $x \in X_{2}^{0}$, each dynamic process $\left(w_{m}: m \in\{0\} \cup \mathbb{N}\right)$ starting at $w_{0}=x$ and satisfying $\forall_{m \in\{0\} \cup \mathbb{N}}\left\{w_{m+1} \in T\left(w_{m}\right)\right\}$ satisfies $\forall_{m \in\{0\} \cup \mathbb{N}}\left\{w_{m+1} \in Q_{2, \Omega ; T}\left(w_{m}\right)\right\}$.

Then assertion (i) is of the form

(i') $\{w\}=T(w)$.

Proof. The proof will be broken into two steps.

Step 1. Assume that assumptions (a)-(g) hold.

Define the relation $\leq_{X_{2}^{0}}$ on $X_{2}^{0}$ as follows:

$$
\forall_{u_{1}, u_{2} \in X_{2}^{0}}\left\{u_{2} \leq_{X_{2}^{0}} u_{1} \Longleftrightarrow u_{2} \in Q_{2, \Omega ; T}\left(u_{1}\right)\right\}
$$

Using analogous argumentation as in the proof of Theorem 4.1 we obtain that $\left(X_{2^{\prime}}^{0} \leq{ }_{X_{2}^{0}}\right)$ is a partial quasiordering space; for each $v \in X_{2}^{0}$, the set $\left\{u \in X_{2}^{0}: u \leq_{X_{2}^{0}} v\right\}$ is complete; each decreasing sequence $\left(w_{m}: m \in \mathbb{N}\right)$ in $X_{2}^{0}$ is 2-Cauchy; and $X_{2}^{0}$ contains at least one minimal element.

Let $w \in X_{2}^{0}$ be a minimal element of $X_{2}^{0}$. Hence, $w \in Q_{2, \Omega ; T}(w)$ and this gives $w \in$ $T(w)$ and $\forall_{\alpha \in \mathcal{A}}\left\{\omega_{\alpha}(w)+\varepsilon_{\alpha} J_{\alpha}(w, w) \varliminf_{H} \omega_{\alpha}(w)\right\}$. Consequently, $w \in T(w)$ and $w \in D_{\Omega} \cap X_{2}^{0}$. Therefore, (i) holds.

Step 2. Assume that assumptions (a)-(h) hold.

By Step $1, w \in T(w)$ where $w \in X_{2}^{0}$ is a minimal element of $X_{2}^{0}$. We prove that $\{w\}=$ $T(w)$. Otherwise, there exists $w^{\prime} \in T(w)$ satisfying $w^{\prime} \neq w$. However, by (h), for each dynamic process $\left(w_{m}: m \in\{0\} \cup \mathbb{N}\right)$ starting at $w_{0}=w$ and such that $w_{1}=w^{\prime}$ we have $\left\{w^{\prime} \in\right.$ $\left.Q_{2, \Omega ; T}(w)\right\}$. Hence it follows that the points $w^{\prime}$ and $w$ are in the relation $w^{\prime} \leq_{X_{2}^{0}} w$. This gives, by minimality of $w, w^{\prime}=w$. This is impossible. Therefore, (i') holds.

Remark 4.6. (a) A special case of condition (g) is a condition $\left(\mathrm{g}^{\prime}\right)$ defined by

$\left(\mathrm{g}^{\prime}\right)$ for each $(x, \alpha) \in X_{2}^{0} \times \mathcal{A}$, the map $\omega_{\alpha}(\cdot)+\varepsilon_{\alpha} J_{\alpha}(x, \cdot): T(x) \cap X_{2}^{0} \rightarrow H \cup\{+\infty\}$ is $\left(T(x) \cap X_{2}^{0}, X\right)$-lsc and, for each $x \in X_{2}^{0}$, the set $Q_{2, \Omega ; T}(x)$ is nonempty.

(b) If $\partial=p$, then a special case of condition ( $\mathrm{g})$ is a condition $\left(\mathrm{g}^{\prime \prime}\right)$ defined by

$\left(\mathrm{g}^{\prime \prime}\right)$ for each $(x, \alpha) \in X \times \mathcal{A}$, the map $\omega_{\alpha}(\cdot)+\varepsilon_{\alpha} p_{\alpha}(x, \cdot): T(x) \rightarrow H \cup\{+\infty\}$ is $(T(x), X)$-lsc and, for each $x \in X$, the set $Q_{p \Omega ; T}(x)$ is nonempty.

Definition 4.7. A family $\Omega=\left\{\omega_{\alpha}: X \rightarrow H \cup\{+\infty\}, \alpha \in A\right\}$ is called an entropy of a set-valued dynamic system $(X, T)$ if $\forall_{\alpha \in \mathcal{A}} \forall_{x \in X} \forall_{y \in T(x)}\left\{\varepsilon_{\alpha} J_{\alpha}(x, y) \leq_{H} \omega_{\alpha}(x)-\omega_{\alpha}(y)\right\}$ or $\forall_{\alpha \in \mathcal{A}} \forall_{x \in X} \exists_{y \in T(x)}\left\{\varepsilon_{\alpha} J_{\alpha}(x, y) \preceq_{H} \omega_{\alpha}(x)-\omega_{\alpha}(y)\right\}$. A dynamic system $(X, T)$ is called dissipative if it has an entropy $\Omega$. One says that a family $\Omega$ is lsc if, for each $\alpha \in \mathcal{A}, \omega_{\alpha}$ is lsc.

The notion of a dissipative map in metric space was introduced in [4]. 
Remark 4.8. By Definition 4.3, Remarks 4.4 and 4.6, and Definition 4.7, we see that we established, in particular, the variational principle of Ekeland type for not necessarily lsc families $\Omega$ and endpoint and fixed point theorem of Caristi type for dissipative set-valued dynamic systems with not necessarily lsc entropies $\Omega$. Consequently, our results are original in the literature.

\section{Examples and Comparisons of Our Results with the Well-Known Ones}

We provide some examples to illustrate the concepts introduced so far.

First, we give the example of 2 -family. Let $L$ be an ordered normed space with cone $H \subset L$, let the family $D=\left\{p_{\alpha}: X \times X \rightarrow L, \alpha \in \mathcal{A}\right\}$ be a $D$-family, and let $(X, D)$ be a Hausdorff cone uniform space, with a cone $H$, containing at least two different points.

Example 5.1. Let $W \subset X$, containing at least two different points, be arbitrary and fixed and let $\left\{c_{\alpha}\right\}_{\alpha \in \mathscr{A}} \subset H$ satisfy $\forall_{\alpha \in \mathcal{A}}\left\{0 \prec_{H} c_{\alpha}\right\}$. Then the family $\mathcal{\partial}=\left\{J_{\alpha}: X \times X \rightarrow L, \alpha \in \mathcal{A}\right\}$, defined by $J_{\alpha}(x, y)=0$ if $x=y \in W$ and $J_{\alpha}(x, y)=c_{\alpha}$ if $x \neq y \vee x=y \notin W, x, y \in X$, and $\alpha \in \mathcal{A}$, is a 2-family on $X$.

Indeed, condition (21) obviously holds. Clearly $\forall_{\alpha \in \mathcal{A}} \forall_{x, y, z \in X}\left\{J_{\alpha}(x, y) \leq_{H} J_{\alpha}(x, z)+\right.$ $\left.J_{\alpha}(z, y)\right\}$, therefore condition (22) holds. For proving that (23) holds we assume that the sequences $\left\{x_{m}\right\}$ and $\left\{y_{m}\right\}$ in $X$ satisfy (2.5) and (2.6). Then, in particular, (2.6) yields

$$
\forall_{\alpha \in \mathscr{A}} \forall_{0<\varepsilon_{\alpha}<\left\|c_{\alpha}\right\|} \exists_{m_{0}=m_{0}\left(\alpha, \varepsilon_{\alpha}\right) \in \mathbb{N}} \forall_{m} \geqslant m_{0}\left\{\left\|J_{\alpha}\left(x_{m}, y_{m}\right)\right\|<\varepsilon_{\alpha}<\left\|c_{\alpha}\right\|\right\} .
$$

By (5.1) and definition of 2 , denoting $m^{\prime}=\min \left\{m_{0}\left(\alpha, \varepsilon_{\alpha}\right): \alpha \in \mathcal{A}\right\}$, we conclude that

$$
\exists_{a \in W} \forall_{m} \geqslant m^{\prime}\left\{\left(x_{m}=y_{m}=a\right)\right\}
$$

From (5.1) and (5.2), we get $\forall_{\alpha \in \mathcal{A}} \forall_{0<\varepsilon_{\alpha}<\left\|c_{\alpha}\right\|} \exists_{m^{\prime} \in \mathbb{N}} \forall_{m} \geqslant m^{\prime}\left\{\left\|p_{\alpha}\left(x_{m}, y_{m}\right)\right\|=0<\varepsilon_{\alpha}\right\}$. The result is that the sequences $\left\{x_{m}\right\}$ and $\left\{y_{m},\right\}$ satisfy (2.7). Therefore, property (23) holds.

Example 5.2 illustrates a fixed point version of Theorem 4.5; we show that, for set-valued dynamic system, assumptions (a)-(g) are satisfied and assertion (i) holds but assumption (h) is not satisfied and assertion (i') does not hold.

Example 5.2. Let $(L,\|\cdot\|), L=\mathbb{R}^{2}$, be a real normed space. Then $H=\{(x, y) \in L: x, y \geqslant 0\}$ is a regular solid cone and let $(X, D)$ be a cone metric space (see [53]) with a cone $H$ where $X=$ $[0,1] \subset \mathbb{R}, P=\{p\}$ and $p: X \times X \rightarrow L$ is a cone metric of the form $p(x, y)=(|x-y|, 2|x-y|)$, $x, y \in X$.

Let $W=(1 / 2,1]$ and let $J: X \times X \rightarrow L$ be of the form

$$
J(x, y)=\left\{\begin{array}{ll}
(0,0), & \text { if } x=y \in W, \\
(2,2), & \text { if } x \neq y \vee x=y \notin W,
\end{array} \quad x, y \in X\right.
$$

By Example 5.1, the family $\partial=\{J\}$ is a 2 -family. We see that $X_{2}^{0}=(1 / 2,1] \neq \emptyset$. 
Let $\varepsilon \in(0, \infty)$ be arbitrary and fixed. Defining $\omega: X \rightarrow L$ as follows:

$$
\omega(x)= \begin{cases}\varepsilon \cdot(0,0) & \text { for } x=1, \\ \varepsilon \cdot(2,2) & \text { for } x \in\left[0, \frac{1}{3}\right) \cup\left(\frac{2}{3}, 1\right), \\ \varepsilon \cdot(3,3) & \text { for } x \in\left[\frac{1}{3}, \frac{2}{3}\right]\end{cases}
$$

we observe that $\forall_{x \in X}\left\{0 \preceq_{H} \omega(x)\right\}$ and $D_{\Omega}=\operatorname{dom}(\omega) \neq \emptyset$.

Let $T: X \rightarrow 2^{X}$ be of the form

$$
T(x)= \begin{cases}\left(0, \frac{1}{2}\right) \cup\left(\frac{1}{2}, 1\right] & \text { if } x=0, \\ \{1\} & \text { if } x \in\left(0, \frac{1}{2}\right) \cup\left(\frac{1}{2}, 1\right), \\ \{0,1\} & \text { if } x=\frac{1}{2}, \\ \left\{\frac{3}{4}, 1\right\} & \text { if } x=1 .\end{cases}
$$

We see that assumptions (a)-(f) of Theorem 4.5 are satisfied.

We prove that $(\mathrm{g})$ holds. To aim this, let $x \in X_{2}^{0}=(1 / 2,1]$. We consider two cases

Case 1. If $x \in(1 / 2,1)$, then $Q_{2, \Omega ; T}(x)=\{1\}$. Indeed, then $T(x)=\{1\}$ and

$$
\begin{aligned}
\omega(1)+\varepsilon J(x, 1) & =\varepsilon(0,0)+\varepsilon(2,2)=\varepsilon(2,2) \\
& \leq_{H} \begin{cases}\varepsilon(2,2)=\omega(x) & \text { if } x \in\left(\frac{2}{3}, 1\right), \\
\varepsilon(3,3)=\omega(x) & \text { if } x=\left(\frac{1}{2}, \frac{2}{3}\right]\end{cases}
\end{aligned}
$$

which gives $Q_{2, \Omega ; T}(x)=\{1\}$. Therefore, for each $x \in(1 / 2,1)$, the set $Q_{2, \Omega ; T}(x)$ is nonempty and closed in $X$.

Case 2. If $x=1$, then $Q_{J, \Omega ; T}(1)=\{1\}$. Indeed, $T(x)=\{3 / 4,1\}$ and $\omega(1)+\varepsilon J(1,1)=$ $\varepsilon(0,0)+\varepsilon(0,0)=\varepsilon(0,0) \preceq_{H} \varepsilon(0,0)=\omega(1)$. Hence, $1 \in Q_{2, \Omega ; T}(1)$. We see that $3 / 4 \notin Q_{2, \Omega ; T}(1)$. Indeed, if $3 / 4 \in Q_{2, \Omega ; T}(1)$, then $\omega(3 / 4)+\varepsilon J(1,3 / 4) \preceq_{H} \omega(1)$. On the other hand, by Case 1 , it follows that $\omega(1)+\varepsilon J(3 / 4,1) \leq_{H} \omega(3 / 4)$. Consequently, $\omega(3 / 4)+\varepsilon J(1,3 / 4) \leq_{H} \omega(1) \prec_{H} \omega(1)+$ $\varepsilon J(3 / 4,1) \preceq_{H} \omega(3 / 4)$ which is impossible. Hence, $Q_{2, \Omega ; T}(1)=\{1\}$. Therefore, $Q_{2, \Omega ; T}(1)$ is a nonempty closed set.

Now, we show that assumption (h) does not hold. Otherwise, suppose that, for each $x \in X_{2}^{0}=(1 / 2,1]$, each dynamic process $\left(w_{m}: m \in\{0\} \cup \mathbb{N}\right)$ starting at $w_{0}=x$ and satisfying $\forall_{m \in\{0\} \cup \mathbb{N}}\left\{w_{m+1} \in T\left(w_{m}\right)\right\}$ satisfies $\forall_{m \in\{0\} \cup \mathbb{N}}\left\{w_{m+1} \in Q_{2, \Omega ; T}\left(w_{m}\right)\right\}$. Then, in particular, for $x=1$, 
a dynamic process $\left(w_{m}: m \in\{0\} \cup \mathbb{N}\right)$ starting at $w_{0}=1$ such that $w_{1}=3 / 4$ and $w_{m}=1$ for each $m \geqslant 2$, satisfies $\forall_{m \in\{0\} \cup \mathbb{N}}\left\{w_{m+1} \in Q_{2, \Omega ; T}\left(w_{m}\right)\right\}$. Hence,

$$
\omega\left(\frac{3}{4}\right)+\varepsilon J\left(1, \frac{3}{4}\right)=\omega\left(w_{1}\right)+\varepsilon J\left(w_{0}, w_{1}\right) \preceq_{H} \omega\left(w_{0}\right)=\omega(1)
$$

holds. On the other hand, if $x=3 / 4$, then a dynamic process $\left(w_{m}: m \in\{0\} \cup \mathbb{N}\right)$ starting at $w_{0}=3 / 4$ such that $w_{m}=1$ for each $m \geqslant 1$, also satisfies $\forall_{m \in\{0\} \cup \mathbb{N}}\left\{w_{m+1} \in Q_{2, \Omega ; T}\left(w_{m}\right)\right\}$. Hence,

$$
\omega(1)+\varepsilon J\left(\frac{3}{4}, 1\right)=\omega\left(w_{1}\right)+\varepsilon J\left(w_{0}, w_{1}\right) \leq_{H} \omega\left(w_{0}\right)=\omega\left(\frac{3}{4}\right)
$$

By (5.7) and (5.8), $\omega(3 / 4)+\varepsilon J(1,3 / 4) \preceq_{H} \omega(1) \prec_{H} \omega(1)+\varepsilon J(3 / 4,1) \preceq_{H} \omega(3 / 4)$ which is impossible. Therefore, $(\mathrm{h})$ does not hold.

We proved that there exists $w=1 \in D_{\Omega} \cap X_{2}^{0}=(1 / 2,1]$ such that $w=1 \in T(1)=$ $\{3 / 4,1\}$. Of course, (h) does not hold and $w=1$ is not the endpoint of $T$ in $X$.

Example 5.3 illustrates an endpoint version of Theorem 4.5; we show that, for a setvalued dynamic system, assumptions (a)-(h) are satisfied and assertion (i') holds.

Example 5.3. Let $L, H,(X, p), X, p: X \times X \rightarrow L, W=(1 / 2,1], 2=\{J\}$ and $\Omega=\{\omega\}$ be such as in Example 5.2. Let $T: X \rightarrow 2^{X}$ be defined by

$$
T(x)= \begin{cases}\left(0, \frac{1}{2}\right) \cup\left(\frac{1}{2}, 1\right] & \text { if } x=0, \\ \{1\} & \text { if } x \in\left(0, \frac{1}{2}\right) \cup\left(\frac{1}{2}, 1\right], \\ \{0,1\} & \text { if } x=\frac{1}{2} .\end{cases}
$$

By considerations analogous to those for Example 5.2, we prove that assumptions (a)(g) are satisfied.

We show that assumption (h) also holds. Indeed, let $x \in X_{2}^{0}=(1 / 2,1]$ be arbitrary and fixed. Then each dynamic process $\left(w_{m}: m \in\{0\} \cup \mathbb{N}\right)$ starting at $w_{0}=x$ and satisfying $\forall_{m \in\{0\} \cup \mathbb{N}}\left\{w_{m+1} \in T\left(w_{m}\right)\right\}$ is of the following form.

Case 1. $w_{0}=x \in(1 / 2,1)$ and, for each $m \geqslant 1, w_{m}=1$;

Case 2. For each $m \in\{0\} \cup \mathbb{N}, w_{m}=1$.

Since

$$
\begin{aligned}
& \omega\left(w_{1}\right)+\varepsilon J\left(w_{0}, w_{1}\right)=\omega(1)+\varepsilon J(x, 1)=\varepsilon(0,0)+\varepsilon(2,2)=\varepsilon(2,2) \\
& \unlhd_{H} \begin{cases}\varepsilon(2,2)=\omega(x)=\omega\left(w_{0}\right) & \text { if } x \in\left(\frac{2}{3}, 1\right), \\
\varepsilon(3,3)=\omega(x)=\omega\left(w_{0}\right) & \text { if } x=\left(\frac{1}{2}, \frac{2}{3}\right],\end{cases}
\end{aligned}
$$


thus, in Cases 1 and $2, \forall_{m \in\{0\} \cup \mathbb{N}}\left\{w_{m+1} \in Q_{2, \Omega ; T}\left(w_{m}\right)\right\}$. We proved that, there exists $w=1 \in$ $X_{2}^{0}=(1 / 2,1]$ such that $w=1 \in T(1)=\{1\}$, that is, $w=1$ is the endpoint of $T$ in $X$.

Remark 5.4. There exist examples of cone uniform spaces $(X, D)$ and the maps $T$ that Theorem 4.5 holds simultaneously for some $2 \neq D$ (see, Example 5.2; then $X=[0,1]$ and $\left.X_{2}^{0}=(1 / 2,1]\right)$ and for $\partial=P$ (see Example 5.5, then $\left.X=X_{2}^{0}=[0,1]\right)$. However, in general, this does not hold (see, e.g., Examples 5.6 and 5.7).

Example 5.5. Let $L, H,(X, D), X, p: X \times X \rightarrow L$ and $T: X \rightarrow 2^{X}$ be as in Example 5.2. Let $\varepsilon \in(0, \infty)$ be arbitrary and fixed and let $\Omega=\{\omega\}$, where $\omega: X \rightarrow L$ is of the form

$$
\omega(x)= \begin{cases}\varepsilon \cdot(0,0) & \text { for } x=1, \\ \varepsilon \cdot(4,4) & \text { for } x \in[0,1) \backslash\left\{\frac{1}{4}, \frac{1}{2}, \frac{3}{4}\right\} \\ \varepsilon \cdot(2,2) & \text { for } x=\frac{1}{4} \\ \varepsilon \cdot(6,6) & \text { for } x=\frac{1}{2} \\ \varepsilon \cdot(1,1) & \text { for } x=\frac{3}{4} .\end{cases}
$$

Assuming that $2=p$, by considerations analogous to those for Example 5.2, we prove that assumptions (a)-(f) of Theorem 4.5 are satisfied. Now we show that (g) holds. Indeed, let $x \in X_{p}^{0}=X$. Then the following cases hold.

Case 1. If $x=0$, then $T(0)=(0,1] \backslash\{1 / 2\}$ and we have the following. (a) for $y=1 / 4 \in T(0)$, we calculate that $\omega(1 / 4)+\varepsilon p(0,1 / 4)=\varepsilon(2,2)+\varepsilon(1 / 4,1 / 2)=\varepsilon(9 / 4,5 / 2) \preceq_{H} \varepsilon(4,4)=$ $\omega(0)$; (b) for $y=3 / 4 \in T(0)$, we calculate $\omega(3 / 4)+\varepsilon p(0,3 / 4)=\varepsilon(1,1)+\varepsilon(3 / 4,3 / 2)=$ $\varepsilon(7 / 4,5 / 2) \leq_{H} \varepsilon(4,4)=\omega(0)$; (c) for $y=1 \in T(0)$, we calculate $\omega(1)+\varepsilon p(0,1)=\varepsilon(0,0)+$ $\varepsilon(1,2)=\varepsilon(1,2) \preceq_{H} \varepsilon(4,4)=\omega(0)$; (d) for each $y \in T(0) \backslash\{1 / 4,3 / 4,1\}$, we calculate $\omega(y)+\varepsilon p(0, y)=\varepsilon(4,4)+\varepsilon(y, 2 y)>_{H} \varepsilon(4,4)=\omega(0)$. Consequently, $Q_{\partial, \Omega ; T}(0)=\{1 / 4,3 / 4,1\}$ is nonempty and closed in $X$.

Case 2. If $x=1 / 2$, then $T(1 / 2)=\{0,1\}$ and we have the following. (a) for $y=0 \in T(1 / 2)$, we get $\omega(0)+\varepsilon p(1 / 2,0)=\varepsilon(4,4)+\varepsilon(1 / 2,1)=\varepsilon(9 / 2,5) \leq_{H} \varepsilon(6,6)=\omega(1 / 2) ;(b)$ for $y=1 \in T(1 / 2)$, we obtain $\omega(1)+\varepsilon p(1 / 2,1)=\varepsilon(0,0)+\varepsilon(1 / 2,1)=\varepsilon(1 / 2,1) \leq_{H} \varepsilon(6,6)=\omega(1 / 2)$. Consequently, $Q_{2, \Omega ; T}(1 / 2)=\{0,1\}$ is nonempty and closed in $X$.

Case 3. If $x \in(0,1 / 2) \cup(1 / 2,1)$, then $T(x)=\{1\}$ and we have

$$
\begin{aligned}
\omega(1)+\varepsilon p(x, 1)= & \varepsilon(0,0)+\varepsilon(1-x, 2(1-x)) \\
& = \begin{cases}\varepsilon\left(\frac{3}{4}, \frac{3}{2}\right) \preceq_{H} \varepsilon(2,2)=\omega\left(\frac{1}{4}\right) & \text { if } x=\frac{1}{4}, \\
\varepsilon(1-x, 2(1-x)) \varliminf_{H} \varepsilon(4,4)=\omega(x) & \text { if } x \in(0,1) \backslash\left\{\frac{1}{2}, \frac{1}{4}, \frac{3}{4}\right\}, \\
\varepsilon\left(\frac{1}{4}, \frac{1}{2}\right) \preceq_{H} \varepsilon(1,1)=\omega\left(\frac{3}{4}\right) & \text { if } x=\frac{3}{4} .\end{cases}
\end{aligned}
$$


Consequently, for each $x \in(0,1 / 2) \cup(1 / 2,1)$ the set $Q_{2, \Omega ; T}(x)=\{1\}$ is nonempty and closed in $X$.

Case 4. If $x=1$, then the set $Q_{2, \Omega ; T}(1)=\{1\}$ is nonempty and closed in $X$.

We proved that assumption (g) holds. Assumption (h) does not hold (see Example 5.2).

It is worth noticing that 2 -families of generalized pseudodistances are very useful and important tools for investigations in cone uniform spaces; for details, see Examples 5.6 and 5.7 below.

Example 5.6. Let $L, H,(X, D), X$ and $p: X \times X \rightarrow L$ be as in Example 5.2.

Let $W=(1 / 2,1]$ and let $J: X \times X \rightarrow L$ be of the form

$$
J(x, y)=\left\{\begin{array}{ll}
(0,0) & \text { if } x=y \in W, \\
(2,2) & \text { if } x \neq y \vee x=y \notin W,
\end{array} \quad x, y \in X .\right.
$$

The family $\partial=\{J\}$ is, by Example 5.1, a 2 -family and $X_{2}^{0}=(1 / 2,1] \neq \emptyset$.

Let $\varepsilon \in(0, \infty)$ be arbitrary and fixed. Defining $\omega: X \rightarrow L$ by the formula

$$
\omega(x)= \begin{cases}\varepsilon \cdot(0,0) & \text { for } x=1 \\ \varepsilon \cdot(2,2) & \text { for } x \in\left[0, \frac{1}{3}\right) \cup\left(\frac{2}{3}, 1\right), \\ \varepsilon \cdot(3,3) & \text { for } x \in\left[\frac{1}{3}, \frac{2}{3}\right]\end{cases}
$$

we see that $\forall_{x \in X}\left\{0 \preceq_{H} \omega(x)\right\}$ and $D_{\Omega}=\operatorname{dom}(\omega) \neq \emptyset$.

Let $T: X \rightarrow 2^{X}$ be of the form

$$
T(x)= \begin{cases}\left\{\frac{1}{2}\right\} & \text { if } x=0, \\ \left(\frac{1}{2}, 1\right] & \text { if } x \in\left(0, \frac{1}{2}\right), \\ \{0\} & \text { if } x=\frac{1}{2}, \\ {\left[0, \frac{1}{2}\right] \cup\{1\}} & \text { if } x \in\left(\frac{1}{2}, 1\right), \\ \{0,1\} & \text { if } x=1 .\end{cases}
$$

Assumptions (a)-(f) of Theorem 4.5 hold. Also (g) holds. Indeed, we have the following cases. 
Case 1. If $x \in(1 / 2,1)$, then $T(x)=[0,1 / 2] \cup\{1\}$. We see that $T(x) \cap X_{2}^{0}=\{1\}$ and

$$
\begin{aligned}
\omega(1)+\varepsilon J(x, 1) & =\varepsilon(0,0)+\varepsilon(2,2)=\varepsilon(2,2) \\
& \preceq_{H} \begin{cases}\varepsilon(2,2)=\omega(x) & \text { if } x \in\left(\frac{2}{3}, 1\right), \\
\varepsilon(3,3)=\omega(x) & \text { if } x=\left(\frac{1}{2}, \frac{2}{3}\right] .\end{cases}
\end{aligned}
$$

This gives $Q_{2, \Omega ; T}(x)=\{1\}$. Hence, for each $x \in(1 / 2,1)$, the set $Q_{2, \Omega ; T}(x)$ is nonempty and closed in $X$.

Case 2. If $x=1$, then $T(1)=\{0,1\}$ and we see that $T(x) \cap X_{2}^{0}=\{1\}$ and $\omega(1)+\varepsilon J(1,1)=$ $\varepsilon(0,0)+\varepsilon(0,0)=\varepsilon(0,0) \preceq_{H} \varepsilon(0,0)=\omega(1)$. Consequently, $Q_{2, \Omega ; T}(1)=\{1\}$ is nonempty and closed in $X$.

Therefore, assumptions (a)-(g) of Theorem 4.5 are satisfied and there exists $w=1 \in$ $X_{2}^{0}=(1 / 2,1]$ such that $1 \in T(1)=\{0,1\}$. Thus Theorem 4.5 holds for $\partial \neq D$. It is easy to show that $(\mathrm{h})$ does not hold.

Example 5.7. Let $L, H, X, p, D=\{p\},(X, D)$ and $T: X \rightarrow 2^{X}$ be such as in Example 5.6. However, let $\partial=p$ (by Remark 2.7, it is $\partial$-family on $X$ ). Of course, $X_{2}^{0}=X \neq \emptyset$. Thus assumptions (a)-(c), (e) and (f) of Theorem 4.5 are satisfied.

Suppose that there exists $\Omega=\{\omega\}$ satisfying $(\mathrm{d})$ and $(\mathrm{g})$. Then, for $x=0$, the set $Q_{2, \Omega ; T}(0) \subset\{1 / 2\}=T(0)$ is nonempty and closed, so $\omega(1 / 2)+p(0,1 / 2) \leq_{H} \omega(0)$. On the other hand, $Q_{2, \Omega ; T}(1 / 2) \subset\{0\}=T(1 / 2)$ is also nonempty and closed, that is, $\omega(0)+p(1 / 2,0) \leq_{H} \omega(1 / 2)$. Hence, $\omega(1 / 2)+p(0,1 / 2) \leq_{H} \omega(0) \prec_{H} \omega(0)+p(1 / 2,0) \leq_{H} \omega(1 / 2)$. This is impossible. Thus (g) does not hold, and we may not use Theorem 4.5 when $\partial=p$.

Example 5.8 illustrates Theorem 4.5 for single-valued dynamic systems.

Example 5.8. Let $(L,\|\cdot\|)$ where $L=\mathbb{R}^{2}, H=\{(x, y) \in L: x, y \geqslant 0\},(X, p)$ be a cone metric space with a cone $H$ where $X=\mathbb{R}, D=\{p\}$ and $p: X \times X \rightarrow L$ is a cone metric of the form $p(x, y)=(|x-y|, 2|x-y|), x, y \in X$.

Let $W=\mathbb{R} \backslash \mathbb{Z}$ and let $J: X \times X \rightarrow L$ be of the form

$$
J(x, y)=\left\{\begin{array}{ll}
(0,0) & \text { if } x=y \in W, \\
(2,2) & \text { if } x \neq y \vee x=y \notin W,
\end{array} \quad x, y \in X\right.
$$

By Example 5.1, $\partial=\{J\}$ is a 2 -family. Moreover, we see that $X_{\partial}^{0}=W \neq \emptyset$. Let $\varepsilon>0$ be arbitrary and fixed. Define $\omega: X \rightarrow L$ as follows: 
Fixed Point Theory and Applications

$$
\omega(x)= \begin{cases}\varepsilon \cdot(4 n, 4 n) & \text { for } x \in(-(2 n+1),-2 n), n \geqslant 3, \\ \varepsilon \cdot(3 n, 6 n) & \text { for } x \in(-2 n,-(2 n-1)), n \in \mathbb{N}, \\ \varepsilon \cdot(4,4) & \text { if } x \in(-3,-2), \\ \varepsilon \cdot(6,6) & \text { if } x \in(-5,-4) \cup(-1,3) \backslash\left\{-\frac{1}{2}, 0, \frac{1}{2}, 1,2\right\}, \\ \varepsilon \cdot(0,0) & \text { if } x=\left\{\frac{1}{2}\right\} \\ \varepsilon \cdot(2,2) & \text { if } x \in\left\{-\frac{1}{2}\right\} \cup \mathbb{Z}, \\ \varepsilon \cdot(4 n, 8 n) & \text { if } x \in(n, n+1), n \geqslant 3 .\end{cases}
$$

We observe that $\forall_{x \in X}\left\{0 \preceq_{H} \omega(x)\right\}$ and $D_{\Omega}=\operatorname{dom}(\omega) \neq \emptyset$. Let $T: X \rightarrow X$ be defined as follows:

$$
T(x)= \begin{cases}-2 n+1 & \text { if } x=-(2 n+1), n \in \mathbb{N}, \\ \frac{(-4 n+3)}{2} & \text { if } x \in(-(2 n+1),-2 n), n \in \mathbb{N} \\ -2 n+2 & \text { if } x=-2 n, n \in \mathbb{N}, \\ \frac{(-4 n+5)}{2} & \text { if } x \in(-2 n,-(2 n-1)), n \in \mathbb{N} \\ \frac{1}{2 n+1} & \text { if } x \in[-1,2) \\ \frac{(4 n-3)}{2} & \text { if } x=2 n, n \in \mathbb{N}, \\ \frac{2 n}{2 n} & \text { if } x=2 n+1, n \in \mathbb{N}, \\ \frac{(4 n-1)}{2} & \text { if } x \in(2 n+1,2 n+2), n \in \mathbb{N} .\end{cases}
$$

Now we prove that (g) holds. Let $x \in X_{\partial}^{0}=W$ be arbitrary and fixed and consider the following eleven cases.

Case 1. For $x \in(-(2 n+1),-2 n), n \geqslant 3$, we have $T(x)=(-4 n+3) / 2 \in X_{2}^{0}$. Consequently, we obtain (a) $\omega((-4 n+3) / 2)+\varepsilon J(x,(-4 n+3) / 2)=\varepsilon(6,6)+\varepsilon(2,2)=\varepsilon(8,8) \leq_{H} \varepsilon(12,12)=\omega(x)$ if $n=3$; (b) $\omega((-4 n+3) / 2)+\varepsilon J(x,(-4 n+3) / 2)=\varepsilon(4(n-1), 4(n-1))+\varepsilon(2,2)=\varepsilon(4 n-2,4 n-$ 2) $\preceq_{H} \varepsilon(4 n, 4 n)=\omega(x)$ if $n>3$. Hence, $Q_{2, \Omega ; T}(x)=\{(-4 n+3) / 2\}$.

Case 2. For $x \in(-5,-4)$, we have $T(x)=(-4 \cdot 2+3) / 2=-5 / 2 \in(-3,-2) \subset X_{2}^{0}$ and $\omega(-5 / 2)+$ $\varepsilon J(x,-5 / 2)=\varepsilon(4,4)+\varepsilon(2,2)=\varepsilon(6,6) \preceq_{H} \varepsilon(6,6)=\omega(x)$, which gives $Q_{2, \Omega ; T}(x)=\{-5 / 2\}$.

Case 3. For $x \in(-3,-2)$, we have $T(x)=(-4 \cdot 1+3) / 2=-1 / 2 \in X_{2}^{0}$ and $\omega(-1 / 2)+\varepsilon J(x,-1 / 2)=$ $\varepsilon(2,2)+\varepsilon(2,2)=\varepsilon(4,4) \preceq_{H} \varepsilon(4,4)=\omega(x)$, which gives $Q_{2, \Omega ; T}(x)=\{-1 / 2\}$.

Case 4. For $x \in(-2 n,-(2 n-1)), n \geqslant 2$, we have $T(x)=(-4 n+5) / 2 \in(-2(n-1),-(2(n-$ 1) - 1)) $\subset X_{2}^{0}$ and $\omega((-4 n+5) / 2)+\varepsilon J(x,(-4 n+5) / 2)=\varepsilon(3(n-1), 6(n-1))+\varepsilon(2,2)=$ $\varepsilon(3 n-1,6 n-4) \leq_{H} \varepsilon(3 n, 6 n)=\omega(x)$, which gives $Q_{2, \Omega ; T}(x)=\{(-4 n+5) / 2\}$. 
Case 5. For $x=(-2,-1)$, we have $T(x)=1 / 2 \in X_{2}^{0}$ and $\omega(1 / 2)+\varepsilon J(x, 1 / 2)=\varepsilon(0,0)+\varepsilon(2,2)=$ $\varepsilon(2,2) \leq_{H} \varepsilon(3,6)=\omega(x)$, which gives $Q_{2, \Omega ; T}(x)=\{1 / 2\}$.

Case 6. For $x=(-1,3) \backslash\{0,1 / 2,1,2\}$, we have $T(x)=1 / 2 \in X_{2}^{0}$ and

$$
\omega\left(\frac{1}{2}\right)+\varepsilon J\left(x, \frac{1}{2}\right)= \begin{cases}\varepsilon(0,0)+\varepsilon(2,2) \preceq_{H} \varepsilon(6,6)=\omega(x) & \text { if } x \neq-\frac{1}{2} \\ \varepsilon(0,0)+\varepsilon(2,2) \preceq_{H} \varepsilon(2,2)=\omega(x) & \text { if } x=-\frac{1}{2}\end{cases}
$$

which gives $Q_{2, \Omega ; T}(x)=\{1 / 2\}$.

Case 7. For $x=1 / 2$, we have $Q_{2, \Omega ; T}(x)=\{1 / 2\}$.

Case 8. For $x \in(4,5)$, we have $T(x)=5 / 2 \in(2,3) \subset X_{2}^{0}$ and $\omega(5 / 2)+\varepsilon J(x, 5 / 2)=\varepsilon(6,6)+$ $\varepsilon(2,2)=\varepsilon(8,8) \preceq_{H} \varepsilon(16,32)=\omega(x)$, which gives $Q_{2, \Omega ; T}(x)=\{5 / 2\}$.

Case 9. For $x \in(2 n, 2 n+1), n \geqslant 3$, we have $T(x)=(4 n-3) / 2 \in(2(n-1), 2(n-1)+1) \subset X_{2}^{0}$ and $\omega((4 n-3) / 2)+\varepsilon J(x,(4 n-3) / 2)=\varepsilon(4(2(n-1)), 8(2(n-1)))+\varepsilon(2,2)=\varepsilon(8 n-6,16 n-$ $14) \preceq_{H} \varepsilon(4(2 n), 8(2 n))=\omega(x)$, which gives $Q_{2, \Omega ; T}(x)=\{(4 n-3) / 2\}$.

Case 10. For $x=(3,4)$, we have $T(x)=3 / 2 \in(1,2) \subset X_{2}^{0}$ and $\omega(3 / 2)+\varepsilon J(x, 3 / 2)=\varepsilon(6,6)+$ $\varepsilon(2,2)=\varepsilon(8,8) \leq_{H} \varepsilon(12,24)=\omega(x)$, which gives $Q_{2, \Omega ; T}(x)=\{3 / 2\}$.

Case 11. For $x \in(2 n+1,2 n+2), n \geqslant 2$, we have $T(x)=(4 n-1) / 2 \in(2(n-1)+1,2(n-1)+2) \subset X_{2}^{0}$ and $\omega((4 n-1) / 2)+\varepsilon J(x,(4 n-1) / 2)=\varepsilon(4(2(n-1)+1), 8(2(n-1)+1))+\varepsilon(2,2)=\varepsilon(8 n-2,16 n-$ 6) $\preceq_{H} \varepsilon(8 n, 16 n) \leq_{H} \varepsilon(4(2 n+1), 8(2 n+1))=\omega(x)$, which gives $Q_{2, \Omega ; T}(x)=\{(4 n-1) / 2\}$.

Consequently, for each $x \in X_{2}^{0}, Q_{2, \Omega ; T}(x)$ is a nonempty and closed subset of $X$.

We proved that there exists $w=1 / 2 \in X_{2}^{0}$ such that $T(1 / 2)=1 / 2$, that is, $w=1 / 2$ is a fixed point of $T$ in $X$.

Remark 5.9. In general, $\left(X_{2}^{0}, T\right)$ is not a dynamic system; indeed, in Example 5.6 we have that $X_{2}^{0}=(1 / 2,1], T\left(X_{2}^{0}\right)=[0,1 / 2] \cup\{1\}$ and $T\left(X_{2}^{0}\right) \nsubseteq X_{2}^{0}$. It is worth noticing that in Example 5.8, $T(E)=\{1 / 2\} \subset X_{2}^{0}$ for $E=\{-1,0,1\} \subset X \backslash X_{2}^{0}$.

Recall that a map $f: X \rightarrow(-\infty,+\infty]$ is proper if its effective domain, $\operatorname{dom}(f)=\{x:$ $f(x)<+\infty\}$, is nonempty. A map $f: X \rightarrow[-\infty,+\infty]$ is lower semicontinuous on $X$ (written: lsc) if the set $\{x \in X: f(x) \leqslant r\}$ is a closed subset in $X$ for each $r \in \mathbb{R}$.

In the literature, the several variants of the variational principle of Ekeland type for lsc maps and fixed point and endpoint theorem of Caristi type for dissipative single-valued and set-valued dynamic systems with lsc entropies in metric and uniform spaces and in metric and uniform spaces with generalized distances are given and various techniques and methods of investigations (notably based on maximality principle) are presented. However, in all these papers assumptions about lower semicontinuity are essential.

Now, we present comparisons between our results and the well-known ones.

We may read, respectively, the results of Mizoguchi [5] and Aubin and Siegel [4], concerning the existence of endpoints of dissipative set-valued dynamic systems with lsc entropy in uniform and metric spaces, respectively, as follows. 
Theorem 5.10 (Mizoguchi [5, Theorems 1 and 2]). Let X be a Hausdorff complete uniform space with a family $\left\{d_{\alpha}: \alpha \in \mathcal{A}\right\}$ of pseudometrics inducing the topology of $X, \omega: X \rightarrow(-\infty,+\infty]$ be a map which is proper lsc and bounded from below and $\left\{\varepsilon_{\alpha}, \alpha \in \mathcal{A}\right\}$ be a family of finite positive numbers.

Endpoint Theorem of Caristi Type. Assume that a set-valued dynamic system $(X, T)$ has the property: $\forall_{\alpha \in \mathcal{A}} \forall_{x \in X} \forall_{y \in T(x)}\left\{\varepsilon_{\alpha} d_{\alpha}(x, y) \leqslant \omega(x)-\omega(y)\right\}$. Then $T$ has an endpoint in $X$.

Variational Principle of Ekeland Type. For any $w_{0} \in X$, there exists $w \in X$ such that: $\forall_{x \in X \backslash\{w\}} \exists_{\beta \in \mathcal{A}}\left\{\omega(w)<\omega(x)+\varepsilon_{\beta} d_{\beta}(w, x)\right\}$, and $\forall_{\alpha \in \mathcal{A}}\left\{\omega(w) \leqslant \omega\left(w_{0}\right)-\varepsilon_{\alpha} d_{\alpha}\left(w, w_{0}\right)\right\}$.

Theorem 5.11 (Aubin and Siegel [4]). Let $(X, d)$ be a complete metric space and let $(X, T)$ be a set-valued dynamic system. Let $\omega: X \rightarrow(-\infty,+\infty]$ be a map which is proper lsc and bounded from below. Assume that $\forall_{x \in X} \forall_{y \in T(x)}\{d(x, y) \leqslant \omega(x)-\omega(y)\}$. Then $T$ has an endpoint in $X$.

The results of Feng and Liu [6] concerning the existence of fixed points and endpoints of dissipative set-valued maps with lsc entropy in metric spaces, of Caristi type, may be read, respectively, as follows.

Theorem 5.12 (Feng and Liu [6, Theorem 4.2 and Corollary 4.3]). Let $(X, d)$ a complete metric space, $(X, T)$ a set-valued dynamic system, $\omega: X \rightarrow \mathbb{R}$ a bounded from below and lsc map, and $\eta:[0, \infty) \rightarrow[0, \infty)$ a nondecreasing, continuous, and subadditive map and such that $\eta^{-1}(\{0\})=$ $\{0\}$. If $\forall_{x \in X} \exists_{y \in T(x)}\{\eta(d(x, y)) \leqslant \omega(x)-\omega(y)\}$, then there exists $w \in X$ such that $w \in T(w)$. If $\forall_{x \in X} \forall y \in T(x)\{\eta(d(x, y)) \leqslant \omega(x)-\omega(y)\}$, then there exists $w \in X$ such that $T(w)=\{w\}$.

In Example 5.13 we show that even for $\partial=p$, Theorem 4.5 is different from Theorems $5.10,5.11$, and 5.12 .

Example 5.13. Let $L=\mathbb{R}, H=[0, \infty), X=[0,1] \subset \mathbb{R}, p=\{d\}, d(x, y)=|x-y|, x, y \in X$. Let $T: X \rightarrow 2^{X}$ be as in Example 5.3.

Suppose that there exists a proper lsc on $X$ and bounded from below map $\omega_{1}: X \rightarrow L$ satisfying

$$
\forall_{x \in X} \forall_{y \in T(x)}\left\{d(x, y) \leqslant \omega_{1}(x)-\omega_{1}(y)\right\}
$$

Let $\eta: H \rightarrow H$ be such as in Theorem 5.12 and suppose that there exists a proper lsc on $X$ and bounded from below map $\omega_{2}: X \rightarrow L$ satisfying

$$
\forall_{x \in X} \forall_{y \in T(x)}\left\{\eta(d(x, y)) \leqslant \omega_{2}(x)-\omega_{2}(y)\right\}
$$

Observe that $0<1 / 2=d(1 / 2,0) \leqslant \omega_{1}(1 / 2)-\omega_{1}(0)$. Moreover, the condition $\eta^{-1}(\{0\})=$ $\{0\}$ implies that $0<\eta(d(1 / 2,0)) \leqslant \omega_{2}(1 / 2)-\omega_{2}(0)$. Therefore,

$$
\omega_{i}(0)<\omega_{i}\left(\frac{1}{2}\right), \quad i=1,2 .
$$

On the other hand, for $x=0$ and for each $y \in T(0)=(0,1 / 2) \cup(1 / 2,1]$, by (5.21) and (5.22), we derive, respectively, that $0<y=d(0, y) \leqslant \omega_{1}(0)-\omega_{1}(y)$ and $0<\eta(d(0, y)) \leqslant \omega_{2}(0)-\omega_{2}(y)$. This gives $T(0) \subset\left\{y \in X: \omega_{i}(y) \leqslant \omega_{i}(0)\right\}, i=1,2$. Also, we see that $0 \in\left\{y \in X: \omega_{i}(y) \leqslant\right.$ 
$\left.\omega_{i}(0)\right\}, i=1,2$. Moreover, (5.23) implies that $1 / 2 \notin\left\{y \in X: \omega_{i}(y) \leqslant \omega_{i}(0)\right\}, i=1,2$. Since $X=[0,1]=T(0) \cup\{0,1 / 2\}$, we conclude that $\left\{y \in X: \omega_{i}(y) \leqslant \omega_{i}(0)\right\}=T(0) \cup\{0\}, i=1,2$, which gives that the set $\left\{y \in X: \omega_{i}(y) \leqslant \omega_{i}(0)\right\}=[0,1 / 2) \cup(1 / 2,1], i=1,2$, is open in $X$. Therefore, for each $i=1,2$, the lsc map $\omega_{i}$ on $X$ has the property that $\left\{y \in X: \omega_{i}(y) \leqslant c\right\}$ is not closed for $c=\omega_{i}(0)$. This is impossible. Consequently, for such $T$, we may not apply Theorems 5.10-5.12 (there does not exist $\omega$ which is lsc on $X$ ).

Definition 5.14 (Vályi [34, page 130]). Let $(Y, \mho)$ be a topological vector space ordered by the closed cone $K$ and $(X, \mathcal{U})$ a Hausdorff complete uniform space. The map $\omega: X \rightarrow Y \cup\{+\infty\}$ is called (strongly) lower semicontinuous, lsc, if for every $x \in \operatorname{dom}(\omega)$ and $(e \in \operatorname{int}(K)) e \in K \backslash\{0\}$ there is a $U \in \mathcal{U}$ such that $(x, y) \in U$ implies that $\omega(y) \geq_{K} \omega(x)-e$.

Let $\omega: X \rightarrow Y \cup\{+\infty\}$ be lsc and bounded from below and let $d: X \times X \rightarrow Y \cup\{+\infty\}$ satisfy:

(i) $0 \leq_{K} d(x, y)$ and $d(x, y)=0$ if $x=y$;

(ii) $d(x, z) \leq_{K} d(x, y)+d(y, z)$;

(iii) for each $x \in X$, the map $y \rightarrow d(x, y)$ is lsc on $X$.

Let $\leq_{d, \omega}$ be a partial quasiordering on $X$ defined as follows: $x \leq_{d, \omega} y$ if $x=y$ or $d(x, y) \leq_{K} \omega(x)-\omega(y)$. For details, see [34, page 130].

The results of Vályi [34], Caristi [2], Ekeland [3], and Jachymski [7], concerning dissipative single-valued dynamic systems with lsc entropies, may be read, respectively, as follows.

Theorem 5.15 (Vályi [34, Theorems 5 and 6]). Let $(X, \mathcal{U})$ be a Hausdorff uniform space; let $(Y, \mho)$ be a weakly sequentially complete topological vector space ordered by the closed normal cone K; let $d: X \times X \rightarrow Y$ satisfy (i) and (ii); let $\omega: X \rightarrow Y \cup\{+\infty\}$ be bounded from below. Assume that (a) the map $\omega$ is continuous (lsc); (b) for each $x \in X$, the map $y \rightarrow d(x, y)$ is continuous (lsc); and (c) for each $U \in U$ there is $a V \in \mathcal{U}$ such that $d(x, y) \in V$ implies that $(x, y) \in U$.

Fixed Point Theorem of Caristi Type). Assume that a map $T: X \rightarrow X$ has the property $\forall_{x \in X}\left\{d(x, T(x)) \leq_{K} \omega(x)-\omega(T(x))\right\}$. Then $T$ has a fixed point in $X$.

Variational Priciple of Ekeland Type. For each $x \in \operatorname{dom}(\omega)$ there exists $w \in \operatorname{dom}(\omega)$ such that $x \leq_{d, \omega} w$ and $w$ is maximal in $\left\{y \in X: x \leq_{d, \omega} y\right\}$ (i.e., $\forall_{y \in X \backslash\{w\}}\left\{\omega(y)_{K} \not \omega(w)-d(w, y)\right\}$ and $\left.\omega(w) \leq_{K} \omega(x)-d(x, w)\right)$.

Theorem 5.16. Let $(X, d)$ be a complete metric space and let $\omega: X \rightarrow(-\infty,+\infty]$ be a map which is proper lsc and bounded from below.

(Caristi [2]). Let $T: X \rightarrow X$. If

$$
\forall_{x \in X}\{d(x, T(x)) \leqslant \omega(x)-\omega(T(x))\}
$$

then there exists $w \in X$ such that $T(w)=w$.

(Ekeland [3]). For every $\varepsilon>0$ and for every $x_{0} \in \operatorname{dom}(\omega)$, there exists $u \in X$ such that: (i) $\omega(u)+\varepsilon d\left(x_{0}, u\right) \leqslant \omega\left(x_{0}\right)$; and (ii) $\forall_{x \in X \backslash\{u\}}\{\omega(u)<\omega(x)+\varepsilon d(x, u)\}$. 
Theorem 5.17 (Jachymski [7, Theorem 6]). Let $(X, d)$ be a complete metric space, $T: X \rightarrow X$, $\omega: X \rightarrow \mathbb{R}$ a nonnegative lsc map on $X$, and $\eta:[0, \infty) \rightarrow[0, \infty)$ a nondecreasing and subadditive map, continuous at 0 and such that $\eta^{-1}(\{0\})=\{0\}$. If $\forall_{x \in X}\{\eta(d(x, T(x)) \leqslant \omega(x)-\omega(T(x))\}$, then there exists $w \in X$ such that $T(w)=w$.

Example 5.18 shows that Theorem 4.5 is different from Theorems 5.15, 5.16, and 5.17.

Example 5.18. Let $L=\mathbb{R}, H=[0, \infty], X=\mathbb{R}, P=\{d\}, d(x, y)=|x-y|$, and $x, y \in X$. Let $T$ : $X \rightarrow X$ be such as in Example 5.8. It is worth noticing that, by Remark 2.7, $\partial=P$ is 2 -family. Suppose that there exists a proper lsc and bounded from below map $\omega_{1}: X \rightarrow(-\infty,+\infty]$ satisfying $\forall_{x \in X}\left\{d(x, T(x)) \leqslant \omega_{1}(x)-\omega_{1}(T(x))\right\}$. Moreover, let $\eta: H \rightarrow H$ be such as in Theorem 5.17 and suppose that there exists a proper lsc on $X$ map $\omega_{2}: X \rightarrow H$ satisfying $\forall_{x \in X}\left\{\eta(d(x, T(x))) \leqslant \omega_{2}(x)-\omega_{2}(T(x))\right\}$. It is clear that

$$
\eta(d(x, T(x)))=0 \quad \text { if } d(x, T(x))=0 .
$$

Let $n_{0} \in \mathbb{N}$ be arbitrary and fixed. We have $0<1=d\left(2 n_{0}, 2 n_{0}+1\right)=d\left(2 n_{0}, T\left(2 n_{0}\right)\right) \leqslant$ $\omega_{1}\left(2 n_{0}\right)-\omega_{1}\left(2 n_{0}+1\right)$ and, by (5.25), $0<\eta\left(d\left(2 n_{0}, 2 n_{0}+1\right)\right)=\eta(d(x, T(x))) \leqslant \omega_{2}\left(2 n_{0}\right)-$ $\omega_{2}\left(2 n_{0}+1\right)$, which gives $\omega_{i}\left(2 n_{0}+1\right)<\omega_{i}\left(2 n_{0}\right), i=1$, 2 . On the other hand, $T\left(2 n_{0}+1\right)=2 n_{0}$, $0<1=d\left(2 n_{0}+1,2 n_{0}\right) \leqslant \omega_{1}\left(2 n_{0}+1\right)-\omega_{1}\left(2 n_{0}\right)$ and, by (5.25), $0<\eta\left(d\left(2 n_{0}+1,2 n_{0}\right)\right) \leqslant$ $\omega_{2}\left(2 n_{0}+1\right)-\omega_{2}\left(2 n_{0}\right)$, which gives $\omega_{i}\left(2 n_{0}\right)<\omega_{i}\left(2 n_{0}+1\right), i=1,2$. This is impossible.

The Banach fixed point theorem may be read as follows.

Theorem 5.19 (Banach [1]). Let $(X, d)$ be a complete metric space and let $T: X \rightarrow X$ be a singlevalued map satisfying the condition

$$
\exists_{0 \leqslant \lambda<1} \forall_{x, y \in X}\{d(T(x), T(y)) \leqslant \curlywedge d(x, y)\} .
$$

Then (i) Thas a unique fixed point $w$ in $X$, and (ii) the sequence $\left\{T^{[m]}(v)\right\}$ converges to $w$ for each $v \in X$.

The maps $T$ satisfying conditions (5.26) and (5.24) are called in literature Banach's contractions and Caristi's maps, respectively. They are essentially different: the map $T$ satisfying (5.26) is continuous and has a unique fixed point while the map $T$ satisfying (5.24) is not necessarily continuous and has a fixed point which is not necessarily unique.

We also illustrate our results in the case when the maps have more than one fixed point (Example 5.20(A)) or one endpoint (Example 5.20(B)).

Example 5.20. Let $(L,\|\cdot\|)$ where $L=\mathbb{R}^{2}, H=\{(x, y) \in L: x, y \geqslant 0\},(X, p)$ be a cone metric space with a cone $H$ where $X=\mathbb{R}, D=\{p\}$ and $p: X \times X \rightarrow L$ is a cone metric of the form $p(x, y)=(|x-y|, 2|x-y|), x, y \in X$.

Let $W_{i}$ and $J_{i}: X \times X \rightarrow L, i=1,2$, be of the form $W_{1}=[0,1 / 4), W_{2}=(3 / 4,1]$,

$$
J_{i}(x, y)=\left\{\begin{array}{ll}
(0,0) & \text { if } x=y \in W_{i} \\
(2,2) & \text { if } x \neq y \vee x=y \notin W_{i},
\end{array} \quad x, y \in X, i=1,2\right.
$$


By Example 5.1, for each $i=1,2, \partial_{i}=\left\{J_{i}\right\}$ is a 2 -family. Moreover, we see that $X_{\partial_{i}}^{0}=W_{i} \neq \emptyset$, $i=1$, 2 . Let $\varepsilon>0$ be arbitrary and fixed. Define $\omega_{i}: X \rightarrow L, i=1,2$, as follows:

$$
\begin{aligned}
& \omega_{1}(x)= \begin{cases}\varepsilon \cdot(0,0) & \text { for } x=0, \\
\varepsilon \cdot(2,2) & \text { for } x \in\left(0, \frac{1}{3}\right) \cup\left(\frac{2}{3}, 1\right], \\
\varepsilon \cdot(3,3) & \text { for } x \in\left[\frac{1}{3}, \frac{2}{3}\right]\end{cases} \\
& \omega_{2}(x)= \begin{cases}\varepsilon \cdot(0,0) & \text { for } x=1, \\
\varepsilon \cdot(2,2) & \text { for } x \in\left[0, \frac{1}{3}\right) \cup\left(\frac{2}{3}, 1\right), \\
\varepsilon \cdot(3,3) & \text { for } x \in\left[\frac{1}{3}, \frac{2}{3}\right] .\end{cases}
\end{aligned}
$$

We observe that $\forall_{x \in X}\left\{0 \leq_{H} \omega_{i}(x)\right\}$ and $D_{\Omega_{i}}=\operatorname{dom}\left(\omega_{i}\right) \neq \emptyset$, where $\Omega_{i}=\left\{\omega_{i}\right\}, i=1,2$.

(A) Let $T_{1}: X \rightarrow 2^{X}$ be defined as follows:

$$
T_{1}(x)= \begin{cases}\{0,1\} & \text { if } x=0, \\ \{0\} & \text { if } x \in\left(0, \frac{1}{4}\right), \\ \left\{\frac{3}{4}\right\} & \text { if } x=\frac{1}{4}, \\ {\left[0, \frac{1}{4}\right]} & \text { if } x \in\left(\frac{1}{4}, \frac{3}{4}\right), \\ \left\{\frac{1}{4}\right\} & \text { if } x=\frac{3}{4}, \\ \{1\} & \text { if } x \in\left(\frac{3}{4}, 1\right), \\ \{0,1\} & \text { if } x=1 .\end{cases}
$$

Step 1. First, we observe that for $\partial_{1}$ and $\Omega_{1}$ assertions (a)-(f) of Theorem 4.5 hold. We prove that $(\mathrm{g})$ holds. We see that $X_{\partial_{1}}^{0}=W_{1}=[0,1 / 4)$ and consider two cases

Case 1. For $x=0$, we have $T_{1}(x)=\{0,1\}$ and we see that $T_{1}(x) \cap X_{\partial_{1}}^{0}=\{0\}$ and $\omega_{1}(0)+$ $\varepsilon J_{1}(0,0)=\varepsilon(0,0)+\varepsilon(0,0) \preceq_{H} \varepsilon(0,0)=\omega_{1}(0)$. Consequently, $Q_{\partial_{1}, \Omega_{1} ; T_{1}}(0) \stackrel{X_{1}}{=}\{0\}$.

Case 2. For $x \in(0,1 / 4)$, we have $T_{1}(x)=\{0\} \subset X_{\partial_{1}}^{0}$ and $\omega_{1}(0)+\varepsilon J_{1}(x, 0)=\varepsilon(0,0)+\varepsilon(2,2)=$ $\varepsilon(2,2) \preceq_{H} \varepsilon(2,2)=\omega_{1}(x)$, which gives $Q_{\partial_{1}, \Omega_{1} ; T_{1}}(x) \stackrel{\text { C }}{=}\{0\}$.

Consequently, for each $x \in X_{\partial_{1}}^{0}, Q_{\partial_{1}, \Omega_{1} ; T_{1}}(x)$ is a nonempty and closed subset of $X$ and there exists $w=0 \in X_{\partial_{1}}^{0}$ such that $w \in T_{1}(0)=\{0,1\}$, that is, $w=0$ is a fixed point of $T_{1}$ in $X$. 
Step 2. We see that for $\partial_{2}$ and $\Omega_{2}$ assertions (a)-(f) of Theorem 4.5 hold. We prove that (g) holds. We see that $X_{\partial_{2}}^{0}=W_{2}=(3 / 4,1]$ and consider two cases.

Case 1. For $x \in(3 / 4,1)$, we have $T_{1}(x)=\{1\} \subset X_{\partial_{2}}^{0}$ and $\omega_{2}(1)+\varepsilon J_{2}(x, 1)=\varepsilon(0,0)+\varepsilon(2,2)=$ $\varepsilon(2,2) \leq_{H}(2,2)=\omega_{2}(x)$, which gives $Q_{\partial_{2}, \Omega_{2} ; T_{1}}(x)=\{1\}$.

Case 2. For $x=1$, we have $T_{1}(x)=\{0,1\}$ and we see that $T_{1}(x) \cap X_{\partial_{2}}^{0}=\{1\}$ and $\omega_{2}(1)+$ $\varepsilon J_{2}(1,1)=\varepsilon(0,0)+\varepsilon(0,0) \leq_{H}(0,0)=\omega_{2}(1)$, which gives $Q_{\partial_{2}, \Omega_{2} ; T_{1}}(1)=\{1\}$.

Consequently, for each $x \in X_{\partial_{2}}^{0}, Q_{\partial_{2}, \Omega_{2} ; T_{1}}(x)$ is a nonempty and closed subset of $X$ and there exists $w=1 \in X_{\partial_{2}}^{0}$ such that $w \in T_{1}(1)=\{0,1\}$, that is, $w=1$ is a fixed point of $T_{1}$ in $X$.

Clearly, (h) does not hold for $\left(\partial_{i}, \Omega_{i}\right), i=1$, 2. Indeed, in Step 1 , if $x=0$, then a dynamic process $\left(w_{m}: m \in\{0\} \cup \mathbb{N}\right)$ starting at $w_{0}=x=0$ and satisfying $\forall_{m \in\{0\} \cup \mathbb{N}}\left\{w_{m+1} \in T_{1}\left(w_{m}\right)\right\}$ such that $w_{1}=1 \in\{0,1\}=T_{1}\left(w_{0}\right)$ and $w_{m}=1$ for $m \geqslant 2$, does not satisfy $\forall_{m \in\{0\} \cup \mathbb{N}}\left\{w_{m+1} \in\right.$ $\left.Q_{2_{1}, \Omega_{1} ; T_{1}}\left(w_{m}\right)\right\}$ since $w_{1}=1 \notin Q_{\alpha_{1}, \Omega_{1} ; T_{1}}\left(w_{0}\right)=\{0\}$. Similarly, in Step 2 , if $x=1$, then a dynamic process $\left(w_{m}: m \in\{0\} \cup \mathbb{N}\right)$ starting at $w_{0}=x=1$ and satisfying $\forall_{m \in\{0\} \cup \mathbb{N}}\left\{w_{m+1} \in T_{1}\left(w_{m}\right)\right\}$ such that $w_{1}=0 \in\{0,1\}=T_{1}\left(w_{0}\right)$ and $w_{m}=0$ for $m \geqslant 2$, does not satisfy $\forall_{m \in\{0\} \cup \mathbb{N}}\left\{w_{m+1} \in\right.$ $\left.Q_{\partial_{2}, \Omega_{2} ; T_{1}}\left(w_{m}\right)\right\}$ since $w_{1}=0 \notin Q_{\partial_{2}, \Omega_{2} ; T_{1}}\left(w_{0}\right)=\{1\}$.

(B) Let $T_{2}: X \rightarrow 2^{X}$ be defined as follows:

$$
T_{2}(x)= \begin{cases}\{0\} & \text { if } x \in\left[0, \frac{1}{4}\right), \\ \left\{\frac{3}{4}\right\} & \text { if } x=\frac{1}{4} \\ {\left[0, \frac{1}{4}\right]} & \text { if } x \in\left(\frac{1}{4}, \frac{3}{4}\right), \\ \left\{\frac{1}{4}\right\} & \text { if } x=\frac{3}{4}, \\ \{1\} & \text { if } x \in\left(\frac{3}{4}, 1\right] .\end{cases}
$$

Step 1. First, we observe that for $\partial_{1}$ and $\Omega_{1}$ assertions (a)-(f) of Theorem 4.5 hold. We prove that $(\mathrm{g})$ and $(\mathrm{h})$ hold. We see that $X_{\partial_{1}}^{0}=W_{1}=[0,1 / 4)$ and consider two cases.

Case 1. For $x=0$, we have $T_{2}(x)=\{0\} \subset X_{\partial_{1}}^{0}$ and $\omega_{1}(0)+\varepsilon J_{1}(0,0)=\varepsilon(0,0)+\varepsilon(0,0) \preceq_{H} \varepsilon(0,0)=$ $\omega_{1}(0)$. Consequently, $Q_{\partial_{1}, \Omega_{1} ; T_{2}}(0)=\{0\}$.

Case 2. For $x \in(0,1 / 4)$, we have $T_{2}(x)=\{0\} \subset X_{2_{1}}^{0}$ and $\omega_{1}(0)+\varepsilon J_{1}(x, 0)=\varepsilon(0,0)+\varepsilon(2,2)=$ $\varepsilon(2,2) \preceq_{H} \varepsilon(2,2)=\omega_{1}(x)$, which gives $Q_{\partial_{1}, \Omega_{1} ; T_{2}}(x)=\{0\}$. is, $(\mathrm{g})$ holds.

Consequently, for each $x \in X_{\partial_{1}}^{0}, Q_{\partial_{1}, \Omega_{1} ; T_{2}}(x)$ is a nonempty and closed subset of $X$, that

For each $x \in X_{\partial_{1}}^{0}$ each dynamic process $\left(w_{m}: m \in\{0\} \cup \mathbb{N}\right)$ starting at $w_{0}=x$ and satisfying $\forall_{m \in\{0\} \cup \mathbb{N}}\left\{w_{m+1} \in T_{2}\left(w_{m}\right)\right\}$ is of the followimg form: (1) for each $m \in\{0\} \cup \mathbb{N}, w_{m}=0$; or $(2) w_{0}=x \in(0,1 / 4)$ and $w_{m}=0$ for $m \geqslant 1$. Therefore, $\forall$ $m \in\{0\} \cup \mathbb{N}\left\{w_{m+1} \in Q_{2_{1}, \Omega_{1} ; T_{2}}\left(w_{m}\right)\right\}$. This gives (h). 

in $X$.

There exists $w=0 \in X_{\partial_{1}}^{0}$ such that $w \in T_{2}(0)=\{0\}$, that is, $w=0$ is an endpoit of $T_{2}$

Step 2. We see that for $\partial_{2}$ and $\Omega_{2}$ assertions (a)-(f) of Theorem 4.5 hold. We prove that $(\mathrm{g})$ and (h) hold. We see that $X_{\partial_{2}}^{0}=W_{2}=(3 / 4,1]$ and consider two cases.

Case 1. For $x \in(3 / 4,1)$, we have $T_{2}(x)=\{1\} \subset X_{\partial_{2}}^{0}$ and $\omega_{2}(1)+\varepsilon J_{2}(x, 1)=\varepsilon(0,0)+\varepsilon(2,2)=$ $\varepsilon(2,2) \leq_{H} \varepsilon(2,2)=\omega_{2}(x)$, which gives $Q_{\partial_{2}, \Omega_{2} ; T_{2}}(x) \stackrel{=}{=}\{1\}$.

Case 2. For $x=1$, we have $T_{2}(x)=\{1\} \subset X_{\partial_{2}}^{0}$ and $\omega_{2}(1)+\varepsilon J_{2}(1,1)=\varepsilon(0,0)+\varepsilon(0,0) \preceq_{H} \varepsilon(0,0)=$ $\omega_{2}(1)$, which gives $Q_{2_{2}, \Omega_{2} ; T_{2}}(1)=\{1\}$.

Consequently, for each $x \in X_{\partial_{2}}^{0}, Q_{\partial_{2}, \Omega_{2} ; T_{2}}(x)$ is a nonempty and closed subset of $X$, that is, (g) holds.

For each $x \in X_{\partial_{2}}^{0}$, each dynamic process $\left(w_{m}: m \in\{0\} \cup \mathbb{N}\right)$ starting at $w_{0}=x$ and satisfying $\forall_{m \in\{0\} \cup \mathbb{N}}\left\{w_{m+1} \in T_{2}\left(w_{m}\right)\right\}$ is of the form: (1) for each $m \in\{0\} \cup \mathbb{N}$ ), $w_{m}=1$; or (2) $w_{0}=x \in(3 / 4,1)$ and $w_{m}=1$ for $m \geqslant 1$. Hence, $\forall_{m \in\{0\} \cup \mathbb{N}}\left\{w_{m+1} \in Q_{2_{2}, \Omega_{2} ; T_{2}}\left(w_{m}\right)\right\}$. This gives (h). in $X$.

There exists $w=1 \in X_{\partial_{2}}^{0}$ such that $w \in T_{2}(1)=\{1\}$, that is, $w=1$ is an endpoint of $T_{2}$

Example 5.21. Let $L, H, X, p, P=\{p\},(X, P)$ and $T: X \rightarrow 2^{X}$ be such as in Example 5.20(A), or (B). However, let $\partial=D$ (by Remark 2.7, it is 2 -family on $X$ ). Of course, $X_{2}^{0}=X \neq \emptyset$. Thus assumptions (a)-(c), (e) and (f) of Theorem 4.5 are satisfied.

Suppose that there exists $\Omega=\{\omega\}$ satisfying (d) and (g). Then, for $x=1 / 4$, the set $Q_{p, \Omega ; T}(1 / 4) \subset\{3 / 4\}=T(1 / 4)$ is nonempty and closed, so $\omega(3 / 4)+p(1 / 4,3 / 4) \preceq_{H} \omega(1 / 4)$. On the other hand, $Q_{p, \Omega ; T}(3 / 4) \subset\{1 / 4\}=T(3 / 4)$ is also nonempty and closed, that is, $\omega(1 / 4)+p(3 / 4,1 / 4) \leq_{H} \omega(3 / 4)$. Hence, $\omega(3 / 4)+p(1 / 4,3 / 4) \leq_{H} \omega(1 / 4) \prec_{L} \omega(1 / 4)+$ $p(3 / 4,1 / 4) \preceq_{H} \omega(3 / 4)$. This is impossible. Thus (g) does not hold, and we may not use Theorem 4.5 when $2=p$.

Finally, we give a remark to end the section.

Remark 5.22. (i) In our investigations the lower semicontinuity assumption is not necessary (see Examples 5.2-5.6 and 5.20).

(ii) The conditions of Caristi-Ekeland type do not hold for maps which have periodic points (see, e.g., [4-29] and references therein). By using 2 -families of generalized pseudodistances we can study even maps which have periodic points (see, e.g., Example 5.6 where $T(0)=\{1 / 2\}$ and $T(1 / 2)=\{0\}$; Example 5.8 where $T(2 n)=$ $2 n+1$ and $T(2 n+1)=2 n, n \in \mathbb{N}$; Example 5.20 where $T(3 / 4)=\{1 / 4\}$ and $T(1 / 4)=$ $\{3 / 4\})$.

(iii) The existence of 2 -families such that $2 \neq D$ is essential (see, e.g., Examples 5.6, 5.7, $5.8,5.20$ and 5.21).

\section{Relations between Generalized Pseudodistances and Distances of Tataru, Kada-Suzuki-Takahashi, Suzuki, Lin-Du, and Vályi}

The aim of this section is to prove that each distance [30-34] is a generalized pseudodistance (see Theorem 6.11). Moreover, we construct the examples (see Examples 6.13, 6.14, and 6.15) 
which show that the converse is not true. This relation betwen generalized pseudodistances and distances [30-34] is important from the point of view of nonlinear analysis.

We will start by definitions and remarks so that this section is self-contained.

Let $X$ be a subset of a Banach space. In 1992, Tataru [30] introduced the distance $p$ : $X \times X \rightarrow[0, \infty)$ (called Tataru's distance) defined by the formula

$$
p(x, y)=\inf \left\{t+\|T(t) x-y\|: t \in \mathbb{R}_{+}\right\}, \quad x, y \in X,
$$

where $\left\{T(t): t \in \mathbb{R}_{+}\right\}$is a strongly continuous semigroup of nonexpansive maps on $X$ defined as follows.

Definition 6.1. Let $X$ be a subset of a Banach space. The family $\{T(t): t \geqslant 0\}$ of maps $T(t)$ : $X \rightarrow X, t \in \mathbb{R}_{+}$, is called a strongly continuous semigroup of nonexpansive maps on $X$ if the following conditions hold:

(sg1) for each $t \in \mathbb{R}_{+}, T(t)$ is a nonexpansive map on $X$;

(sg2) $\forall_{x \in X}\{T(0) x=x\}$;

(sg3) $\forall_{s, t \in \mathbb{R}_{+}}\{T(s+t)=T(s) \circ T(t)\}$;

(sg4) for each $x \in X$, the map $T(\cdot) x$ from $\mathbb{R}_{+}$into $X$ is continuous.

In 1996, Kada et al. [31] introduced the following concept of $w$-distance.

Definition 6.2. Let $X$ be a metric space with metric $d$. A map $p: X \times X \rightarrow[0, \infty)$ is called a $w$-distance on $X$ if it satisfies the following conditions:

(w1) $\forall_{x, y, z \in X}\{p(x, z) \leqslant p(x, y)+p(y, z)\}$;

(w2) $p$ is lsc in its second variable;

(w3) $\forall_{\varepsilon>0} \exists_{\delta>0} \forall_{x, y, z \in X}\{[p(z, x) \leqslant \delta \wedge(z, y) \leqslant \delta] \Rightarrow d(x, y) \leqslant \varepsilon\}$.

In 2001, Suzuki [32] defined $\tau$-distance.

Definition 6.3. Let $X$ be a metric space with metric $d$. A map $p: X \times X \rightarrow[0, \infty)$ is called a $\tau$-distance on $X$ if there exists a map $\eta: X \times[0, \infty) \rightarrow[0, \infty)$ and the following conditions hold:

(S1) $\forall_{x, y, z \in X}\{p(x, z) \leqslant p(x, y)+p(y, z)\}$;

(S2) $\forall_{x \in X} \forall_{t>0}\{\eta(x, 0)=0 \wedge \eta(x, t) \geqslant t\}$ and $\eta$ is concave and continuous in its second variable;

(S3) $\lim _{n \rightarrow \infty} x_{n}=x$ and $\lim _{n \rightarrow \infty} \sup _{m \geqslant n} \eta\left(z_{n}, p\left(z_{n}, x_{m}\right)\right)=0$ imply that $\forall_{w \in X}\{p(w, x) \leqslant$ $\left.\liminf _{n \rightarrow \infty} p\left(w, x_{n}\right)\right\} ;$

(S4) $\left.\lim _{n \rightarrow \infty} \sup _{m \geqslant n} p\left(x_{n}, y_{m}\right)\right)=0$ and $\lim _{n \rightarrow \infty} \eta\left(x_{n}, t_{n}\right)=0$ imply that $\lim _{n \rightarrow \infty} \eta\left(y_{n}, t_{n}\right)$ $=0$;

(S5) $\lim _{n \rightarrow \infty} \eta\left(z_{n}, p\left(z_{n}, x_{n}\right)\right)=0$ and $\lim _{n \rightarrow \infty} \eta\left(z_{n}, p\left(z_{n}, y_{n}\right)\right)=0$ imply that $\lim _{n \rightarrow \infty}$ $d\left(x_{n}, y_{n}\right)=0$. 
Remark 6.4 (see [32, page 442]). Condition (S2) may be replaced by the following condition (S2)':

$(\mathrm{S} 2)^{\prime} \forall_{x \in X}\left\{\inf _{t>0} \eta(x, t)=0\right\}$ and $\eta$ is nondecreasing in its second variable.

Remark 6.5. If $(X, d)$ is a metric space, then the metric $d$ is a $w$-distance (see [31]) and every $w$-distance on $X$ is a $\tau$-distance on $X$ (see [32, Proposition 1, page 443]).

Remark 6.6. If $\{T(t): t>0\}$ is a strongly continuous semigroup of nonexpansive maps on a subset $X$ of a Banach space, then Tataru's distance $p$ on $X$ is also $\tau$-distance on $X$ (see [32, Proposition 2, page 443]).

In 2006, Lin and Du [33] introduced the following concept of $\tau$-function.

Definition 6.7. Let $X$ be a metric space with metric $d$. A map $p: X \times X \rightarrow[0, \infty)$ is called a $\tau$-function on $X$ if the following conditions hold:

(L1) $\forall_{x, y, z \in X}\{p(x, z) \leqslant p(x, y)+p(y, z)\}$;

(L2) if $x \in X$ and $\left\{y_{n}\right\}$ in $X$ with $\lim _{n \rightarrow \infty} y_{n}=y$ and $p\left(x, y_{n}\right) \leqslant M$ for some $M=M(x)>$ 0 , then $p(x, y) \leqslant M$;

(L3) for any sequence $\left\{x_{n}\right\}$ in $X$ with $\lim _{n \rightarrow \infty} \sup \left\{p\left(x_{n}, x_{m}\right): m>n\right\}=0$, if there exists a sequence $\left\{y_{n}\right\}$ in $X$ such that $\lim _{n \rightarrow \infty} p\left(x_{n}, y_{n}\right)=0$, then $\lim _{n \rightarrow \infty} d\left(x_{n}, y_{n}\right)=0$;

(L4) for $x, y, z \in X, p(x, y)=0$, and $p(x, z)=0$ imply that $y=z$.

Remark 6.8. In metric spaces $(X, d)$, every $w$-distance on $X$ is a $\tau$-function on $X$ (see $[33$, Remark 2.1]).

In 1985, Vályi, in assumptions (i), (ii), (5.2) and (5.7) of Theorems 5 and 6 of the paper [34, page 131], introduced and used in uniform spaces the new concept of distance which in this paper we will call by Vályi's distance. In metric spaces, Vályi's distance we may formulate as follows:

Definition 6.9. Let $X$ be a metric space with metric $d$. A map $p: X \times X \rightarrow[0, \infty)$ is called a distance of Vályi on $\mathrm{X}$ if the following conditions hold:

(V1) $\forall_{x, y, z \in X}\{p(x, z) \leqslant p(x, y)+p(y, z)\}$;

(V2) $p$ is lsc in its second variable;

(V3) $\forall x, y \in X\{p(x, y) \geqslant 0 \wedge[p(x, y)=0 \Leftrightarrow x=y]\}$; and

(V4) $\forall_{\varepsilon>0} \exists_{\delta>0} \forall_{x, y \in X}\{p(x, y)<\delta \Rightarrow d(x, y)<\varepsilon\}$.

In the literature there are no studies concerning relations between Vályi's distances [34] and $\tau$-distances [32] and $\tau$-functions [33]. follows.

In metric spaces the generalized pseudodistance (see Definition 2.6(i)) is defined as

Definition 6.10. Let $X$ be a metric space with metric $d$. The map $U: X \times X \rightarrow[0, \infty)$ is said to be a generalized pseudodistance on $X$ if the following two conditions hold:

$$
\text { (U1) } \forall_{x, y, z \in X}\{U(x, z) \leqslant U(x, y)+U(y, z)\} \text {; }
$$


(U2) for any sequence $\left(x_{n}: n \in \mathbb{N}\right)$ in $X$ such that

$$
\lim _{n \rightarrow \infty} \sup _{m>n} U\left(x_{n}, x_{m}\right)=0
$$

if there exists a sequence $\left(y_{n}: n \in \mathbb{N}\right)$ in $X$ satisfying

$$
\lim _{n \rightarrow \infty} U\left(x_{n}, y_{n}\right)=0,
$$

then

$$
\lim _{n \rightarrow \infty} d\left(x_{n}, y_{n}\right)=0
$$

In this section we give the precise relations between generalized pseudodistances and $\tau$-distances of Suzuki [32], $\tau$-functions of Lin and Du [33] and distances of Vályi [34].

By Remarks 6.5 and 6.6 and Definitions 6.3, 6.7, 6.9, and 6.10, the following question arose naturally.

Question 1. Let $(X, d)$ be a metric space and let $p: X \times X \rightarrow[0, \infty)$ be $\tau$-distance of Suzuki on $X$ or $\tau$-function of Lin and Du on X or Vályi's distance on X. Is $p$ a generalized pseudodistance on $X$ ?

In the following theorem we give an affirmative answer to this question.

Theorem 6.11. Let $X$ be a metric space with metric $d$.

(a) If $p: X \times X \rightarrow[0, \infty)$ is a $\tau$-distance, then $p$ is a generalized pseudodistance.

(b) If $p: X \times X \rightarrow[0, \infty)$ is a $\tau$-function, then $p$ is a generalized pseudodistance.

(c) If $p: X \times X \rightarrow[0, \infty)$ is a Vá lyi's distance, then $p$ is a generalized pseudodistance.

Proof. $\quad$ (a) It is clear that (S1) implies $(\mathfrak{U} 1)$.

For proving that $(\mathfrak{U} 2)$ holds we assume that the sequences $\left(x_{n}: n \in \mathbb{N}\right)$ and $\left(y_{n}: n \in \mathbb{N}\right)$ in $X$ satisfy (6.2) and (6.3), that is,

$$
\begin{gathered}
\lim _{n \rightarrow \infty} \sup _{m>n} p\left(x_{n}, x_{m}\right)=0, \\
\lim _{n \rightarrow \infty} p\left(x_{n}, y_{n}\right)=0 .
\end{gathered}
$$

By (6.5), and [32, Lemma 3, page 450], we obtain that $\lim _{n \rightarrow \infty} d\left(x_{n}, y_{n}\right)=0$. Therefore, $(\mathfrak{U 2})$ is satisfied. Consequently, $p$ is a generalized pseudodistance on $X$.

(b) Indeed, conditions (L1) and (L3) imply conditions (U1) and (U2), respectively.

(c) We see that condition (V1) implies (U1).

For proving that $(\mathfrak{U} 2)$ holds, we assume that the sequences $\left(x_{m}: m \in \mathbb{N}\right)$ and $\left(y_{m}:\right.$ $m \in \mathbb{N})$ in $X$ satisfy (6.2) and (6.3). 
By (V4),

$$
\forall_{\varepsilon>0} \exists_{\delta>0} \forall_{x, y \in X}\{p(x, y)<\delta \Longrightarrow d(x, y)<\varepsilon\}
$$

From (6.3),

$$
\forall_{\delta>0} \exists_{n_{0} \in \mathbb{N}} \forall_{n \geqslant n_{0}}\left\{p\left(x_{n}, y_{n}\right)<\delta\right\}
$$

As consequence of (6.6) and (6.7), we get

$$
\forall_{\varepsilon>0} \exists_{n_{0} \in \mathbb{N}} \forall_{n \geqslant n_{0}}\left\{d\left(x_{n}, y_{n}\right)<\varepsilon\right\}
$$

that is, (6.4) holds. Therefore, $(\mathfrak{U} 2)$ is satisfied.

Now we ask the following question.

Question 2. Is converse to Theorem 6.11 true?

The examples constructed in the sequel give a negative answer to this question.

For later use, we begin by constructing a generalized pseudodistance.

Example 6.12. Let $X$ be a metric space with metric $d$. Let the set $E \subset X$, containing at least two different points, be arbitrary and fixed and let $c>0$ satisfy $\delta(E)<c$ where $\delta(E)=$ $\sup \{d(x, y): x, y \in E\}$. Let $U: X \times X \rightarrow[0, \infty)$ be defined by the formula

$$
U(x, y)=\left\{\begin{array}{ll}
d(x, y) & \text { if } E \cap\{x, y\}=\{x, y\}, \\
c & \text { if } E \cap\{x, y\} \neq\{x, y\},
\end{array} \quad x, y \in X .\right.
$$

The map $U$ is a generalized pseudodistance on $X$.

Indeed, it is worth noticing that the condition $(\mathcal{U} 1)$ does not hold only if there exist some $x_{0}, y_{0}, z_{0} \in X$ such that $U\left(x_{0}, z_{0}\right)>U\left(x_{0}, y_{0}\right)+U\left(y_{0}, z_{0}\right)$. This inequality is equivalent to $c>d\left(x_{0}, y_{0}\right)+d\left(y_{0}, z_{0}\right)$ where $U\left(x_{0}, z_{0}\right)=c, U\left(x_{0}, y_{0}\right)=d\left(x_{0}, y_{0}\right)$ and $U\left(y_{0}, z_{0}\right)=d\left(y_{0}, z_{0}\right)$. However, by (6.9), $U\left(x_{0}, z_{0}\right)=c$ gives that there exists $v \in\left\{x_{0}, z_{0}\right\}$ such that $v \notin E, U\left(x_{0}, y_{0}\right)=$ $d\left(x_{0}, y_{0}\right)$ gives $\left\{x_{0}, y_{0}\right\} \subset E$ and $U\left(y_{0}, z_{0}\right)=d\left(y_{0}, z_{0}\right)$ gives $\left\{y_{0}, z_{0}\right\} \subset E$. This is impossible. Therefore, $\forall_{x, y, z \in X}\{U(x, y) \leqslant U(x, z)+U(z, y)\}$, that is, condition (UU1) holds.

For proving that $(\mathfrak{U} 2)$ holds we assume that the sequences $\left(x_{m}: m \in \mathbb{N}\right)$ and $\left(y_{m}: m \in\right.$ $\mathbb{N}$ ) in $X$ satisfy (6.2) and (6.3). Then, in particular, (6.3) yields

$$
\forall_{0<\varepsilon<c} \exists_{m_{0}=m_{0}(\varepsilon) \in \mathbb{N}} \forall_{m \geqslant m_{0}}\left\{U\left(x_{m}, y_{m}\right)<\varepsilon\right\} .
$$

By (6.10) and (6.9), since $\varepsilon<c$, we conclude that

$$
\forall_{m \geqslant m_{0}}\left\{E \cap\left\{x_{m}, y_{m}\right\}=\left\{x_{m}, y_{m}\right\}\right\} .
$$


Fixed Point Theory and Applications

From (6.11), (6.9) and (6.10), we get

$$
\forall_{0<\varepsilon<c} \exists_{m_{0} \in \mathbb{N}} \forall_{m \geqslant m_{0}}\left\{d\left(x_{m}, y_{m}\right)<\varepsilon\right\} .
$$

Therefore, the sequences $\left(x_{m}: m \in \mathbb{N}\right)$ and $\left(y_{m}: m \in \mathbb{N}\right)$ satisfy (6.4). Consequently, property (U2) holds.

The first observation is that there exists a generalized pseudodistance which is not a $\tau$-distance.

Example 6.13. Let $(X, d)$ be a metric space where $X=[0,1]$ and $d: X \times X \rightarrow[0, \infty)$ is of the form $d(x, y)=|x-y|, x, y \in X$. Let $E=(0,1 / 2]$ and, for each $\gamma>0$, define the map $U^{\gamma}: X \times X \rightarrow[0, \infty)$ by

$$
U^{\gamma}(x, y)=\left\{\begin{array}{ll}
d(x, y) & \text { if } E \cap\{x, y\}=\{x, y\}, \\
\frac{1}{2}+\gamma & \text { if } E \cap\{x, y\} \neq\{x, y\},
\end{array} \quad x, y \in X\right.
$$

Using Example 6.12 we find that, for each $\gamma>0$, the map $U^{r}$ is a generalized pseudodistance on $X$.

We show that, for each $\gamma>0$, the map $p^{\gamma}: X \times X \rightarrow[0, \infty)$ of the form

$$
p^{r}(x, y)=U^{r}(x, y), \quad x, y \in X,
$$

is not a $\tau$-distance on $X$.

Indeed, suppose that there exists $\gamma_{0}>0$, such that $p^{\gamma_{0}}$ is a $\tau$-distance on $X$ and consider a sequence $\left(x_{n}: n \in \mathbb{N}\right)$ defined by the formula: $x_{n}=1 / n, n \in \mathbb{N}$. Of course, $\left(x_{n}: n \in \mathbb{N}\right)$ converges in $X$ and

$$
\lim _{n \rightarrow \infty} x_{n}=0
$$

Consequently, $\left(x_{n}: n \in \mathbb{N}\right)$ is a Cauchy sequence on $X$, that is,

$$
\limsup _{n \rightarrow \infty} d\left(x_{n}, x_{m}\right)=0
$$

Next, since $\forall_{n \geqslant 2}\left\{x_{n} \in E\right\}$, by (6.3), we get $\forall_{n, m \in \mathbb{N}}\left\{p^{\gamma_{0}}\left(x_{n}, x_{m}\right)=U^{\gamma_{0}}\left(x_{n}, x_{m}\right)=\right.$ $\left.d\left(x_{n}, x_{m}\right)\right\}$. This and (6.16) give

$$
\limsup _{n \rightarrow \infty} \sup _{m>n} p^{\gamma_{0}}\left(x_{n}, x_{m}\right)=\limsup _{n \rightarrow \infty} d\left(x_{n>n}, x_{m}\right)=0 .
$$

Using now (6.17) and [32, Lemma 3, page 450], we obtain that $\left(x_{n}: n \in \mathbb{N}\right)$ is $p^{r_{0}-C a u c h y}$ (recall that if $X$ is a metric space with metric $d$ and $p$ is a $\tau$-distance on $X$, then a sequence $\left(x_{n}: n \in \mathbb{N}\right)$ in $X$ is called $p$-Cauchy if there exists a function $\eta: X \times[0, \infty) \rightarrow[0, \infty)$ satisfying (S2)-(S5) and a sequence $\left(z_{n}: n \in \mathbb{N}\right)$ in $X$ such that $\lim _{n \rightarrow \infty} \sup _{m \geqslant n} \eta\left(z_{n}, p\left(z_{n}, x_{m}\right)\right)=0$; 
see [32, page 449]). Consequently, there exists a map $\eta: X \times[0, \infty) \rightarrow[0, \infty)$ satisfying (S2)-(S5) and a sequence $\left(z_{n}: n \in \mathbb{N}\right)$ in $X$ such that

$$
\lim _{n \rightarrow \infty} \sup _{m \geqslant n} \eta\left(z_{n}, p^{\gamma_{0}}\left(z_{n}, x_{m}\right)\right)=0
$$

Now (6.17), (6.18), and condition (S3) imply that

$$
\forall_{w \in X}\left\{p^{\gamma_{0}}(w, 0) \leqslant \liminf _{n \rightarrow \infty} p^{\gamma_{0}}\left(w, x_{n}\right)\right\}
$$

However, for $w_{0}=1 / 2 \in X$, since $\forall_{n} \geqslant 2\left\{x_{n} \in E\right\}$ and $w_{0}=1 / 2 \in E$, according to (6.13), we calculate, since $0 \notin E$, that

$$
\begin{aligned}
p^{\gamma_{0}}\left(w_{0}, 0\right) & =p^{\gamma_{0}}\left(\frac{1}{2}, 0\right)=U^{\gamma_{0}}\left(\frac{1}{2}, 0\right)=\frac{1}{2}+\gamma_{0}>\frac{1}{2}=\liminf _{n \rightarrow \infty} d\left(\frac{1}{2}, x_{n}\right) \\
& =\liminf _{n \rightarrow \infty} U^{\gamma_{0}}\left(\frac{1}{2}, x_{n}\right)=\liminf _{n \rightarrow \infty} p^{\gamma_{0}}\left(\frac{1}{2}, x_{n}\right)=\liminf _{n \rightarrow \infty} p^{\gamma_{0}}\left(w_{0}, x_{n}\right)
\end{aligned}
$$

which, by (6.19), is impossible.

Below we include an example which shows that there exists a generalized pseudodistance which is not $\tau$-function.

Example 6.14. Let $X, d$ and $p^{r}: X \times X \rightarrow[0, \infty), \gamma>0$, be defined as in Example 6.13. Of course, for each $\gamma>0, p^{\gamma}$ is a generalized pseudodistance on $X$ (see Example 6.13). Suppose that there exists $\gamma_{0}>0$, such that $p^{\gamma_{0}}$ is $\tau$-function on $X$ and let $\left(y_{n}: n \in \mathbb{N}\right)$ be a sequence given by the formula $y_{n}=1 / n, n \in \mathbb{N}$. We see that

$$
\lim _{n \rightarrow \infty} y_{n}=0
$$

Let $x=1 / 4$. By definition of $\tau$-function, there exists $M=M(x)=1 / 4>0$ such that

$$
\forall_{n \in \mathbb{N}}\left\{p^{\gamma_{0}}\left(x, y_{n}\right)=p^{\gamma_{0}}\left(\frac{1}{4}, \frac{1}{n}\right)=d\left(\frac{1}{4}, \frac{1}{n}\right) \leqslant \frac{1}{4}=M\right\}
$$

Therefore, by (6.21) and (6.22), according (L2), we must have $p^{r_{0}}(1 / 4,0) \leqslant M$. However, by definition of $p^{\gamma_{0}}$, since $0 \notin E$, we get that $p^{\gamma_{0}}(1 / 4,0)=1 / 2+\gamma_{0}>1 / 4=M$. This is absurd.

Finally, it is worth noticing that there exists a generalized pseudodistance which is not Vályi's distance.

Example 6.15. Let $\gamma>0$ be arbitrary and fixed. Then, for a generalized pseudodistance $U^{\gamma}$ constructed in Example 6.13, if $x \notin E$, then we get that $U^{r}(x, x)=1 / 2+\gamma>0$. Therefore, $U^{r}$ does not satisfy (V3). 


\section{References}

[1] S. Banach, "Sur les opérations dans les ensembles abstraits et leurs applications aux équations intégrales," Fundamenta Mathematicae, vol. 3, pp. 133-181, 1922.

[2] J. Caristi, "Fixed point theorems for mappings satisfying inwardness conditions," Transactions of the American Mathematical Society, vol. 215, pp. 241-251, 1976.

[3] I. Ekeland, "On the variational principle," Journal of Mathematical Analysis and Applications, vol. 47, pp. 324-353, 1974.

[4] J.-P. Aubin and J. Siegel, "Fixed points and stationary points of dissipative multivalued maps," Proceedings of the American Mathematical Society, vol. 78, no. 3, pp. 391-398, 1980.

[5] N. Mizoguchi, "A generalization of Brøndsted's results and its applications," Proceedings of the American Mathematical Society, vol. 108, no. 3, pp. 707-714, 1990.

[6] Y. Feng and S. Liu, "Fixed point theorems for multi-valued contractive mappings and multi-valued Caristi type mappings," Journal of Mathematical Analysis and Applications, vol. 317, no. 1, pp. 103-112, 2006.

[7] J. R. Jachymski, "Caristi's fixed point theorem and selections of set-valued contractions," Journal of Mathematical Analysis and Applications, vol. 227, no. 1, pp. 55-67, 1998.

[8] H. Brézis and F. E. Browder, "A general principle on ordered sets in nonlinear functional analysis," Advances in Mathematics, vol. 21, no. 3, pp. 355-364, 1976.

[9] F. E. Browder, "On a theorem of Caristi and Kirk," in Fixed Point Theory and Its Applications, S. Swaminathan, Ed., pp. 23-27, Academic Press, New York, NY, USA, 1976.

[10] A. Brøndsted, “On a lemma of Bishop and Phelps," Pacific Journal of Mathematics, vol. 55, pp. 335-341, 1974.

[11] S. Dančs, M. Hegedús, and P. Medvegyev, "A general ordering and fixed-point principle in complete metric space," Acta Scientiarum Mathematicarum, vol. 46, no. 1-4, pp. 381-388, 1983.

[12] D. Downing and W. A. Kirk, "A generalization of Caristi's theorem with applications to nonlinear mapping theory," Pacific Journal of Mathematics, vol. 69, no. 2, pp. 339-346, 1977.

[13] J.-X. Fang, "The variational principle and fixed point theorems in certain topological spaces," Journal of Mathematical Analysis and Applications, vol. 202, no. 2, pp. 398-412, 1996.

[14] C.-L. Guo, "Fixed point theorems for singlevalued mappings and multivalued mappings on complete metric spaces I," Chinese Journal of Mathematics, vol. 19, no. 1, pp. 31-53, 1991.

[15] C.-L. Guo, "Fixed point theorems for singlevalued mappings and multivalued mappings on complete metric spaces II," Chinese Journal of Mathematics, vol. 19, no. 1, pp. 105-117, 1991.

[16] A. Hamel and A. Löhne, "A minimal point theorem in uniform spaces," in Nonlinear Analysis and Applications: To V. Lakshmikantham on His 80th Birthday, R. P. Agarwal and D. O'Regan, Eds., vol. 1, pp. 577-593, Kluwer Academic Publishers, 2003.

[17] M. A. Khamsi, "Remarks on Caristi's fixed point theorem," Nonlinear Analysis: Theory, Methods $\mathcal{E}$ Applications, vol. 71, no. 1-2, pp. 227-231, 2009.

[18] W. A. Kirk, "Caristi's fixed point theorem and metric convexity," Colloquium Mathematicum, vol. 36, no. 1, pp. 81-86, 1976.

[19] W. A. Kirk, "Caristi's fixed point theorem and the theory of normal solvability," in Fixed Point Theory and Its Applications (Proc. Sem., Dalhousie Univ., Halifax, N.S., 1975), S. Swaminathan, Ed., pp. 109-120, Academic Press, New York, NY, USA, 1976.

[20] A. B. Németh, "Ordered uniform spaces and variational problems," Italian Journal of Pure and Applied Mathematics, no. 16, pp. 183-192, 2004.

[21] A. B. Németh, "Regular ordering and existence of minimum points in uniform spaces and topological groups," Positivity, vol. 8, no. 3, pp. 305-313, 2004.

[22] S. Park, "On extensions of the Caristi-Kirk fixed point theorem," Journal of the Korean Mathematical Society, vol. 19, no. 2, pp. 143-151, 1983.

[23] S. Park, "Equivalent formulations of Ekeland's variational principle for approximate solutions of minimization problems and their applications," in Operator Equations and Fixed Point Theorems, S. P. Singh, et al., Ed., vol. 1, pp. 55-68, MSRI-Korea, 1986.

[24] S. Park and B. G. Kang, "Generalizations of the Ekeland type variational principles," Chinese Journal of Mathematics, vol. 21, no. 4, pp. 313-325, 1993. 
[25] J.-P. Penot, "The drop theorem, the petal theorem and Ekeland's variational principle," Nonlinear Analysis: Theory, Methods \& Applications, vol. 10, no. 9, pp. 813-822, 1986.

[26] A. Petruşel and A. Sîntămărian, "Single-valued and multi-valued Caristi type operators," Publicationes Mathematicae Debrecen, vol. 60, no. 1-2, pp. 167-177, 2002.

[27] M. Turinici, "Maximal elements in topological and generalized metric spaces," Periodica Mathematica Hungarica, vol. 13, no. 3, pp. 259-264, 1982.

[28] Z. Wu, "Equivalent formulations of Ekeland's variational principle," Nonlinear Analysis: Theory, Methods E Applications, vol. 55, no. 5, pp. 609-615, 2003.

[29] C.-K. Zhong, "On Ekeland's variational principle and a minimax theorem," Journal of Mathematical Analysis and Applications, vol. 205, no. 1, pp. 239-250, 1997.

[30] D. Tataru, "Viscosity solutions of Hamilton-Jacobi equations with unbounded nonlinear terms," Journal of Mathematical Analysis and Applications, vol. 163, no. 2, pp. 345-392, 1992.

[31] O. Kada, T. Suzuki, and W. Takahashi, "Nonconvex minimization theorems and fixed point theorems in complete metric spaces," Mathematica Japonica, vol. 44, no. 2, pp. 381-391, 1996.

[32] T. Suzuki, "Generalized distance and existence theorems in complete metric spaces," Journal of Mathematical Analysis and Applications, vol. 253, no. 2, pp. 440-458, 2001.

[33] L.-J. Lin and W.-S. Du, "Ekeland's variational principle, minimax theorems and existence of nonconvex equilibria in complete metric spaces," Journal of Mathematical Analysis and Applications, vol. 323, no. 1, pp. 360-370, 2006.

[34] I. Vályi, "A general maximality principle and a fixed point theorem in uniform space," Periodica Mathematica Hungarica, vol. 16, no. 2, pp. 127-134, 1985.

[35] W. A. Kirk and L. M. Saliga, "The Brézis-Browder order principle and extensions of Caristi's theorem," Nonlinear Analysis: Theory, Methods E Applications, vol. 47, no. 4, pp. 2765-2778, 2001.

[36] H. Lakzian and F. Arabyani, "Some fixed point theorems in cone metric spaces with w-distance," International Journal of Mathematical Analysis, vol. 3, pp. 1081-1086, 2009.

[37] L.-J. Lin and W.-S. Du, "Some equivalent formulations of the generalized Ekeland's variational principle and their applications," Nonlinear Analysis: Theory, Methods \& Applications, vol. 67, no. 1, pp. 187-199, 2007.

[38] L.-J. Lin and W.-S. Du, “On maximal element theorems, variants of Ekeland's variational principle and their applications," Nonlinear Analysis: Theory, Methods \& Applications, vol. 68, no. 5, pp. 1246 $1262,2008$.

[39] S. Park, "On generalizations of the Ekeland-type variational principles," Nonlinear Analysis: Theory, Methods E Applications, vol. 39, no. 7, pp. 881-889, 2000.

[40] N. Shioji, T. Suzuki, and W. Takahashi, "Contractive mappings, Kannan mappings and metric completeness," Proceedings of the American Mathematical Society, vol. 126, no. 10, pp. 3117-3124, 1998.

[41] T. Suzuki, "On Downing-Kirk's theorem," Journal of Mathematical Analysis and Applications, vol. 286, no. 2, pp. 453-458, 2003.

[42] T. Suzuki, "Several fixed point theorems concerning $\tau$-distance," Fixed Point Theory and Applications, vol. 2004, no. 3, pp. 195-209, 2004.

[43] T. Suzuki, "Generalized Caristi's fixed point theorems by Bae and others," Journal of Mathematical Analysis and Applications, vol. 302, no. 2, pp. 502-508, 2005.

[44] T. Suzuki, "Counterexamples on $\tau$-distance versions of generalized Caristi's fixed point theorems," Bulletin of the Kyushu Institute of Technology. Pure and Applied Mathematics, no. 52, pp. 15-20, 2005.

[45] T. Suzuki, "The strong Ekeland variational principle," Journal of Mathematical Analysis and Applications, vol. 320, no. 2, pp. 787-794, 2006.

[46] T. Suzuki, "A definitive result on asymptotic contractions," Journal of Mathematical Analysis and Applications, vol. 335, no. 1, pp. 707-715, 2007.

[47] T. Suzuki, "Subrahmanyam's fixed point theorem," Nonlinear Analysis: Theory, Methods E Applications, vol. 71, no. 5-6, pp. 1678-1683, 2009.

[48] W. Takahashi, "Existence theorems generalizing fixed point theorems for multivalued mappings," in Fixed Point Theory and Applications (Marseille, 1989), J. B. Baillon and M. Théra, Eds., vol. 252 of Pitman Research Notes in Mathematics Series, pp. 397-406, Longman Scientific \& Technical, Essex, UK, 1991.

[49] K. Włodarczyk, R. Plebaniak, and M. Doliński, "Cone uniform, cone locally convex and cone metric spaces, endpoints, set-valued dynamic systems and quasi-asymptotic contractions," Nonlinear Analysis: Theory, Methods \& Applications, vol. 71, no. 10, pp. 5022-5031, 2009.

[50] K. Włodarczyk, R. Plebaniak, and C. Obczyński, "Convergence theorems, best approximation and best proximity for set-valued dynamic systems of relatively quasi-asymptotic contractions in cone uniform spaces," Nonlinear Analysis: Theory, Methods E Applications, vol. 72, no. 2, pp. 794-805, 2010. 
[51] K. Deimling, Multivalued Differential Equations, vol. 1 of de Gruyter Series in Nonlinear Analysis and Applications, Walter de Gruyter, Berlin, Germany, 1992.

[52] G. X.-Z. Yuan, KKM Theory and Applications in Nonlinear Analysis, vol. 218 of Monographs and Textbooks in Pure and Applied Mathematics, Marcel Dekker, New York, NY, USA, 1999.

[53] L.-G. Huang and X. Zhang, "Cone metric spaces and fixed point theorems of contractive mappings," Journal of Mathematical Analysis and Applications, vol. 332, no. 2, pp. 1468-1476, 2007. 